\title{
Determinant Factors and Regulatory Systems for Anthocyanin Biosynthesis in Rice Apiculi and Stigmas
}

Lingzhi Meng ${ }^{1,2 \dagger}$, Changyan $\mathrm{Qi}^{1+}$, Cuihong Wang ${ }^{1}$, Shuai Wang ${ }^{1}$, Chunlei Zhou ${ }^{3}$, Yulong Ren ${ }^{1}$, Zhijun Cheng ${ }^{1}$, Xin Zhang ${ }^{1}$, Xiuping Guo', Zhichao Zhao', Jie Wang ${ }^{1}$, Qibing Lin', Shanshan Zhu', Haiyang Wang',

Zhonghua Wang ${ }^{2^{*}}$, Cailin Lei ${ }^{1^{*}}$ (D) and Jianmin Wan ${ }^{1,3^{*}}$

\begin{abstract}
Anthocyanins cause purple, brown or red colors in various tissues of rice plants, but the specific determinant factors and regulatory systems for anthocyanin biosynthesis in almost all tissues remain largely unknown. In the present study, we mapped and isolated two complementary genes, OsC1 encoding a R2R3-MYB transcriptional factor and OsDFR encoding a dihydroflavonol 4-reductase, which are responsible for the purple coloration of apiculi and stigmas in indica cultivar Xieqingzao by the map-based cloning strategy. We also identified two tissue-specific pigmentation genes, OsPa for apiculi and OsPs for stigmas, by phylogenetic analysis of all anthocyanin biosynthesisassociated bHLH transcriptional factors in maize and rice, CRISPR/Cas9 knockout and transcriptional expression analysis. The OsC1, OsPa and OsPs proteins are all localized in the nucleus while the OsDFR protein is localized in the nucleus and cytoplasm, and the OsC1 and OsDFR genes are preferentially strongly expressed in both purplecolored tissues while the OsPa and OsPs genes are preferentially strongly expressed in apiculi and stigmas, respectively. OsC1 specifically interacts with OsPa or OsPs to activate OsDFR and other anthocyanin biosynthesis genes, resulting in purple-colored apiculi or stigmas. OsC1 itself does not produce color but can produce brown apiculi when functioning together with OsPa. Loss of function of OsDFR alone leads to brown apiculi and strawwhite stigmas. Genotyping and phenotyping of a panel of 176 rice accessions revealed diverse genotypic combinations of OsC1, OsDFR, OsPa and OsPs that enable accurate prediction of their apiculus and stigma pigmentation phenotypes, thus validating the general applicability of the OsC1-OsDFR-OsPa and OsC1-OsDFR-OsPs models to natural populations. Our findings disclosed the biological functions of OsC1, OsPa and OsPs, and shed light on the specific regulatory systems of anthocyanin biosynthesis in apiculi and stigmas, a further step in understanding the regulatory network of anthocyanin biosynthesis in rice.
\end{abstract}

Keywords: Anthocyanin, Apiculus, Oryza sativa, OsC1, OsDFR, OsPa, OsPs, Stigma

\footnotetext{
* Correspondence: zhonghuawang@nwafu.edu.cn; leicailin@caas.cn; wanjianmin@caas.cn

${ }^{\dagger}$ Lingzhi Meng and Changyan Qi contributed equally to this work.

${ }^{2}$ State Key Laboratory of Crop Stress Biology for Arid Areas, College of

Agronomy, Northwest A\&F University, Yangling 712100, Shaanxi, China

'Institute of Crop Sciences, Chinese Academy of Agriculture Sciences/

National Key Facility for Crop Gene Resources and Genetic Improvement,

Beijing 100081, China

Full list of author information is available at the end of the article
}

\section{Springer Open}

(c) The Author(s). 2021 Open Access This article is licensed under a Creative Commons Attribution 4.0 International License, which permits use, sharing, adaptation, distribution and reproduction in any medium or format, as long as you give appropriate credit to the original author(s) and the source, provide a link to the Creative Commons licence, and indicate if changes were made. The images or other third party material in this article are included in the article's Creative Commons licence, unless indicated otherwise in a credit line to the material. If material is not included in the article's Creative Commons licence and your intended use is not permitted by statutory regulation or exceeds the permitted use, you will need to obtain permission directly from the copyright holder. To view a copy of this licence, visit http://creativecommons.org/licenses/by/4.0/. 


\section{Background}

Anthocyanins are a major class of flavonoids that produce colored plant organs. They are involved not only in the pigmentation patterns but also in a wide range of biological functions, such as attraction of pollinators and seed dispersal agents, protection against UV radiation and high light intensity, and defense responses to abiotic and biotic stresses such as cold, drought tolerance and disease (Lin-Wang et al. 2010; Petroni and Tonelli 2011). Moreover, plant anthocyanins reputedly promote human health by protecting against certain cancers, cardiovascular diseases and other chronic disorders (Zhang et al. 2014; Zheng et al. 2019). Therefore, improving our understanding of the anthocyanin biosynthesis and its regulation in rice is a worthwhile objective.

Anthocyanin biosynthesis involves various structural and regulatory genes. The structural genes encode catalytic enzymes, including chalcone synthase (CHS), chalcone isomerase $(\mathrm{CHI})$, flavanone 3-hydroxylase $(\mathrm{F} 3 \mathrm{H})$, flavonoid 3'-hydroxylase (F3'H), flavonoid 3'5'-hydroxylase $\left(\mathrm{F} 3{ }^{\prime} 5^{\prime} \mathrm{H}\right)$, dihydroflavonol 4-reductase (DFR), anthocyanidin synthase (ANS), and UDP-glucose: flavonoid 3O-glucosyltransferase (UFGT) (Zhang et al. 2014). Three transcription factor families, viz. R2R3-MYB, basic helixloop-helix (bHLH), and WD40 repeat protein (WDR), are important regulators of anthocyanin biosynthesis (Xu et al. 2015; Sun et al. 2018; Zheng et al. 2019). In maize, the MYB and bHLH proteins are encoded by two multi-gene families, $C 1 / P l 1$ and $R 1 / B 1$, respectively. A combination of the two types of proteins coordinately activate expression of a series of structural genes. The R1/B1genes, R1, B1, Sn1, Lc1 and Hopi1, whose expressions are tissue-specific, determine distribution of pigments in different tissues (Petroni and Tonelli 2011; Oshima et al. 2019).

Earlier genetic studies of anthocyanin biosynthesis in rice revealed that pigmentation in various tissues was mainly controlled by three factors, $C$ (Chromogen), $A$ (activator) and $P$ (Purple, distributor), where $C$ and $A$ were essentially color-producing genes and $P$ was a tissue-specific regulator of both $C$ and $A$ (Nagao and Takahashi 1956, 1963; Takahashi 1957). Further genetic analyses suggested multiple additional loci that could be responsible for tissue-specific distribution and accumulation of anthocyanins and proanthocyanidins, such as $P l s$ for coleoptile, $P l$ for leaf blade, Psh for leaf sheath, $P i n$ for internode, $P g$ for glume, and $P s$ for stigma (Takahashi 1982; Reddy 1996). Anthocyanin biosynthesisrelated genes in rice were initially isolated by referring to the sequences to known maize orthologs. These included the catalytic enzyme genes OsCHS, OsCHI, OsANS and OsDFR, and putative regulatory genes such as $O s C 1, R a 1 / O s B 1, R b, R c, R a 2$ and $O s B 2$ ( Hu et al. 2000; Reddy et al. 1998, 2007; Druka et al. 2003;
Sakamoto et al. 2001). OsC1, the homolog of maize C1, encodes a R2R3-MYB type transcription factor that is associated with apiculus pigmentation (Zhao et al. 2016). $R c$, the homolog of maize intensifier 1, encodes a bHLH type transcription factor that determines proanthocyanin biosynthesis in pericarps and causes browncolored rice grain. A combination of $R c$ and $R d$ (OsDFR) results in red grain color (Furukawa et al. 2006). kala4 (i.e. OsB2 or S1, Zheng et al. 2019), the homolog of maize $R / B$, encodes a bHLH type transcription factor that is necessary for black grain color. A structural change in the kala4 promoter confers the black pericarp (Oikawa et al. 2015). Sun et al. (2018) proposed the C-SA gene system for regulation of hull pigmentation; here $C 1$ (OsC1) and $A 1$ (OsDFR) collectively determine the color variation, whereas $S 1$ (OsB2) diversifies the pigmentation among tissues. Zheng et al. (2019) also proposed that $O s C 1$, OsDFR and OsRb corresponding to the $C, A$ and $P$ genes coordinately determine anthocyanin biosynthesis in rice leaves. To sum up, even though a handful of R2R3-MYB and bHLH regulators have been identified individually, the comprehensive regulation systems controlling pigmentation in specific tissues and mechanism of anthocyanin biosynthesis in those tissues remain to be determined.

Purple apiculi and stigmas not only attract insects for pollination and animals for seed dispersal, but also serve as visible markers for varietal identification and purification (Chin et al. 2016; Zhao et al. 2016). The $C-A-P$ system was initially established for anthocyanin pigmentation in purple apiculi in rice (Nagao and Takahashi 1956). In this system, $C$ corresponds to OsC1, and $A$ probably corresponds to OsDFR (Zhao et al. 2016; Sun et al. 2018; Zheng et al. 2019); however, the homology of the $P$ gene remains to be determined, let alone its biological nature and function. Purple stigma has also been extensively investigated since the middle of the last century (Takahashi 1957, 1964), and again, the molecular mechanism underlying stigma pigmentation is largely unknown. Oka (1991) showed that pigmentation generally occurred only in plants having $C, A$, and $P$, and that some other tissue-specific genes might also be involved. Han et al. (2006), Chen et al. (2010) and Zhao et al. (2016) fine-mapped the purple stigma gene(s) to the same region as $\mathrm{OsCl}$ on chromosome 6, but $\mathrm{OsCl}$ alone failed to produce a purple stigma. Thus, isolation and characterization of the determinant genes for the pigmentation of apiculi and stigmas present a challenge for a comprehensive dissection of the regulatory network underlying anthocyanin biosynthesis and accumulation in rice.

In the present study we investigated the determinant factors and regulatory system of anthocyanin biosynthesis in rice apiculi and stigmas. Our aims were to 
determine (1) the genetic basis of anthocyanin biosynthesis and accumulation in apiculi and stigmas, and (2) how anthocyanin biosynthesis is specifically regulated in those tissues.

\section{Results}

Phenotypic Characterization of indica Cultivar (cv). Xieqingzao (XQZ)

Purple color in cv. XQZ was present in the apiculi and stigmas at the initial heading stage (Fig. 1a, Fig. S1a, b). The color in both tissues gradually deepened and were quite evident at anthesis (Fig. S1a, b). Japonica cv. Kitaake, displayed no purple color in any tissue throughout its growth cycle (Fig. 1b). The relative anthocyanin contents in the apiculi and stigmas of cv. XQZ were 6.7and 23.4-fold higher than those in cv. Kitaake (Fig. S2), indicating that the apiculus and stigma pigmentation reflected accumulation of anthocyanins.

\section{Genetic Dissection of Purple Apiculi and Stigmas}

$\mathrm{F}_{1}$ individuals derived from the cross $\mathrm{XQZ} \times$ Kitaake exhibited purple apiculi and stigmas similar to XQZ (Fig. S3b). When the apiculus and stigma colors were compared there were three phenotypic classes: ++ (apiculi and stigmas both purple), +' - (apiculi brown, stigmas straw-white) and - (apiculi and stigmas both strawwhite) in the $F_{2}$ population, and the numbers of plants in each fitted a 9:3:4 ratio, respectively (Fig. S3c; Table S1), indicating control by two complementary dominant genes, one of which was responsible for brown apiculi. We tentatively name the two genes as $D$ and $E$, and postulated the genotypes of two parents as DDEE for XQZ, ddee for Kitaake, and $D d E e$ for the $\mathrm{F}_{1}$. The genotypes of three $\mathrm{F}_{2}$ classes were postulated as $D D E E / D d E E / D D E e /$ $D d E e, d d E E / d d E e$, and DDee/Ddee/ddee) (Fig. S3a-c).

\section{Mapping and Candidate Gene Analysis for Purple Apiculi and Stigmas}

We firstly used $1300 \mathrm{~F}_{2}$ individuals with brown apiculi but straw-white stigmas ( $d d E E / d d E e$ ) to map the $D$ gene. The target gene was delimited to a $113 \mathrm{~kb}$ interval flanked by InDel (insertion/deletion) markers $\mathrm{Z} 8$ and Z20 on chromosome $1 \mathrm{~L}$ (Fig. 1c). Within this interval, the gene LOC_Os01g44260 encodes a DFR which catalyzes the conversion of dihydroflavonols to leucoanthocyanidins, a crucial step in the biosynthesis of anthocyanins. OsDFR (i.e. $R d$ ) was known to participate in pigmentation of pericarps, hulls and leaves (Furukawa et al. 2006; Sun et al. 2018; Zheng et al. 2019). Nucleotide sequence alignment between the OsDFR alleles derived from XQZ and Kitaake revealed base substitutions in the second and third exons (Fig. 1d), among which the mutations at sites 462 and 711 were synonymous, the site 301 change led to a I to $\mathrm{V}$ amino acid change, and the site 164 mutation (C to A) caused a premature termination of translation in Kitaake (Fig. 1d). Thus, we postulated that $O s D F R$ to be a candidate for the $D$ gene.

We similarly mapped the $E$ gene using a total of 500 $\mathrm{F}_{2}$ individuals with straw-white apiculi and stigmas (DDee/Ddee/ddee). The target gene was delimited to a $105 \mathrm{~kb}$ interval flanked by InDel markers M1 and M2 on chromosome $6 \mathrm{~S}$ (Fig. 1e). The chromogen gene $\mathrm{OsCl}$ (LOC_Os06g10350) located within this interval encodes a R2R3-MYB transcription factor (Fig. S7a). Subsequent DNA sequencing revealed four bases substitutions ( $C$ to $\mathrm{T}$; $\mathrm{T}$ to $\mathrm{C}$; $\mathrm{C}$ to $\mathrm{A} ; \mathrm{T}$ to $\mathrm{C}$ ) in the third exon of ORF12 between XQZ and Kitaake. The mutations at sites 269, 443 and 716 caused amino acid changes (S to F; P to Q; $\mathrm{V}$ to A) (Fig. 1f). We postulated that $\mathrm{OsC1}$ was a candidate for the $E$ gene.

\section{Functional Validation of OsC1 and OsDFR}

We transformed a $4.8 \mathrm{~kb}$ genomic fragment containing the entire $\mathrm{OsC1}$ allele from XQZ under control of its native promoter into japonica cv. Kitaake with straw-white apiculus and stigma by Agrobacterium-mediated transformation. All positive transformants produced brown apiculi but straw-white stigmas although the pigment appeared much later than in XQZ (Fig. 2a, Fig. S4a, b, d). We then transformed a $5.9 \mathrm{~kb}$ genomic fragment of the entire OsDFR allele from XQZ under control of its native promoter into the above $\mathrm{OsC1}$-transgenic plants. All positive digenic transformants exhibited both purple apiculi and purple stigmas with similar coloring and timing to that in XQZ (Fig. 2b, Fig. S4a, c, d). However, when the same genomic fragment of the functional OsDFR alone was transformed into Kitaake no positive transformant exhibited colored stigmas and apiculi. These results confirmed that complementary genes, $\mathrm{OsC1}$ and $\mathrm{OsDFR}$, were responsible for both purple apiculi and purple stigmas, whereas $\mathrm{OsC1}$ alone gave brown apiculi only in the transgenic plants. Quantitative real-time PCR (qRT-PCR) revealed that $O s D F R$ expression was significantly upregulated with increased $\mathrm{OsC1}$ expression in both single gene $(O s C 1)$ and di-gene $(O s C 1$ and OsDFR) transformants relative to that in Kitaake (Fig. 2c), indicating that OsC1 could activate OsDFR expression. Thus, we deduced that $O s C 1$ is crucial for producing color, and OsDFR plays a role in the OsC1-dependent pathway for purple coloration in rice apiculi and stigmas.

\section{Identification of Tissue-Specific Genes}

Here, we isolated the color-producing gene $O s C 1$ and the activator gene $D F R$, which act coordinately to regulate the purple colors of apiculi and stigmas. Whether some tissue-specific genes were also needed for purple apiculi and stigmas remained to be determined. In maize, the bHLH-type R1/B1 genes were demonstrated 
a
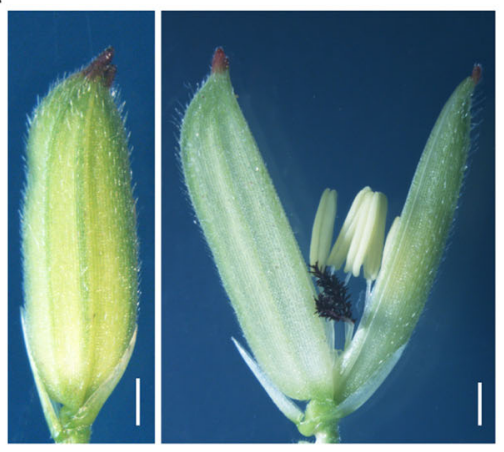

b
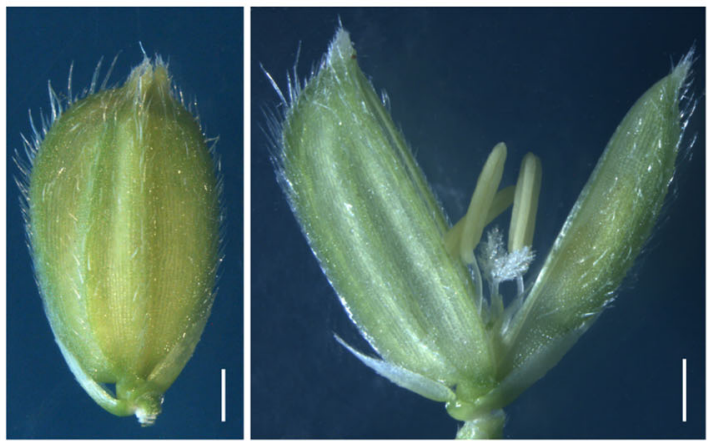

C
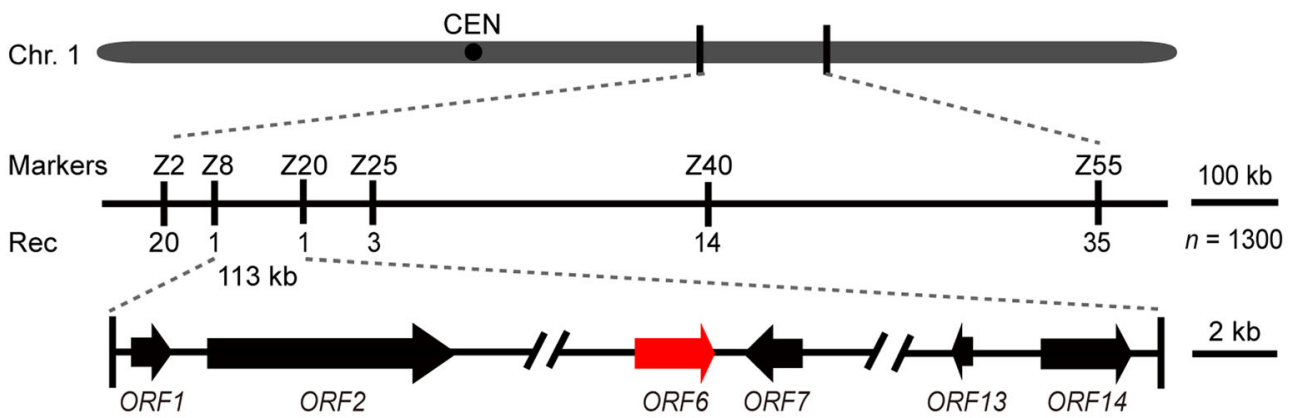

LOC_Os01g44260

d

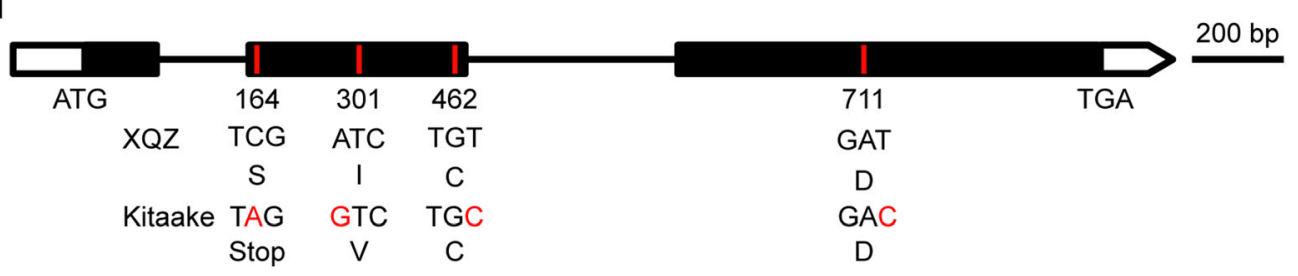

e
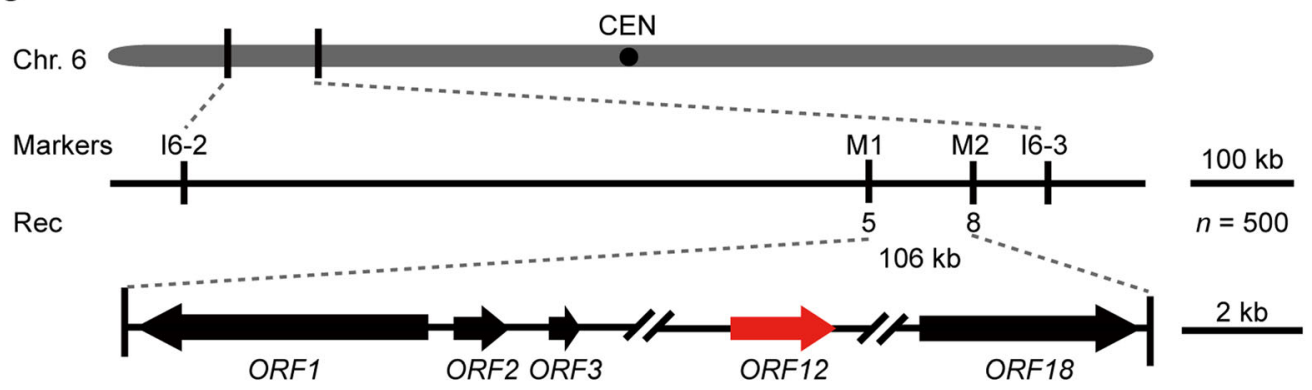

LOC_Os06g10350

f

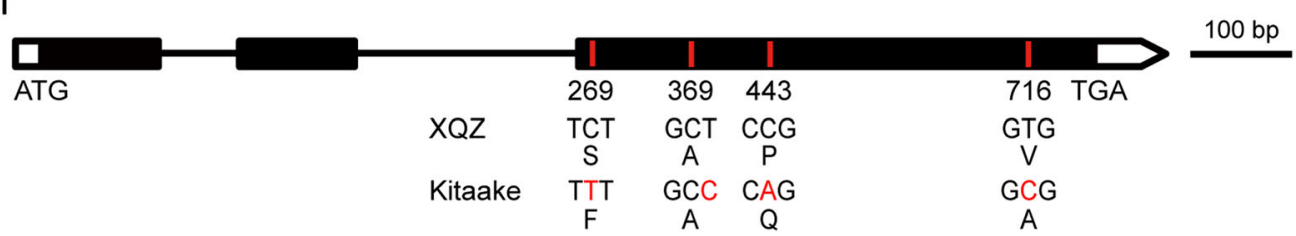

Fig. 1 (See legend on next page.) 
(See figure on previous page.)

Fig. 1 Map-based cloning of OsC1 and OsDFR. a XQZ shows purple apiculus and stigma. Bars, $1 \mathrm{~mm}$. b Straw-white apiculus and stigma in Kitaake. Bars, $1 \mathrm{~mm}$. c Fine mapping of OSDFR. Molecular markers and numbers of recombinants are labeled above and below the filled bars, respectively. Red arrow represents target gene OsDFR. CEN, centromere. Chr. 1, Chromosome 1. Rec, recombinants. d Genomic structure and mutation sites in ORF6 in XQZ and Kitaake. Three exons, two introns, and untranslated regions are indicated by black boxes, black lines, open boxes, respectively. Four bases substitutions in OFR6 between XQZ and Kitaake are highlighted in red. ATG and TGA are the start and stop codons, respectively. e Fine mapping of OsC1. Molecular markers and numbers of recombinants are labeled above and below the filled bars, respectively. The target gene is marked in red. CEN, centromere. Chr. 6, Chromosome 6. Rec, recombinants. f Genomic structure and mutation sites of OsC1 in XQZ and Kitaake. Three exons, two introns, untranslated regions are indicated by black boxes, black lines, open boxes respectively. Four bases substitutions in ORF12 between XQZ and Kitaake are highlighted in red. ATG and TGA are the start and stop codons, respectively

to be tissue-specific and determine the tissue distribution of pigments (Petroni and Tonelli 2011; Oshima et al. 2019). Considering the high synteny of $R / B$ genes in rice and maize (Hu et al. 1996), we performed a phylogenetic analysis of all rice bHLH transcriptional factors (TFs) and all known maize bHLH TFs associated with anthocyanin biosynthesis to identify candidate tissue-specific genes. Nine rice bHLH-type TFs (Fig. S5) were found to be closest to the maize $R$ genes. Among them, $R c, S 1$ and $O s R b$ had been reported to act as tissue-specific genes participating in coloration of pericarps, hulls and leaf blades, respectively (Furukawa et al.
2006; Sun et al. 2018; Zheng et al. 2019), and the other six TFs had unknown functions. We tentatively named these six TFs HLH1 to HLH6 (Table S2).

To determine biological functions of the six TF-encoding genes, we knocked out all of them in the backgrounds of XQZ and the japonica landrace Lijiangxintuanheigu (LTH) using the CRISPR/Cas9 method. LTH possesses purple apiculi, purple stigmas (Fig. S6a, b). Only the HLH1- and HLH2-knockout mutants, i.e. hlh1-1, hlh2-1 and hlh2-2 in the XQZ background, and hlh1-2, hlh1-3, hlh2-3 and hlh2-4 in LTH background, displayed mutant color phenotypes (Fig. 3a-c, Fig. S6a-c). Knockouts of the other four

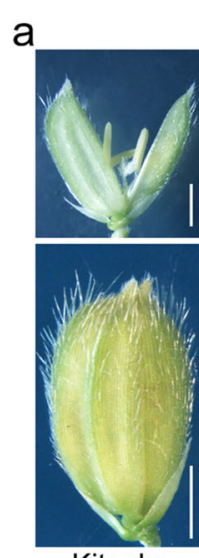

Kitaake
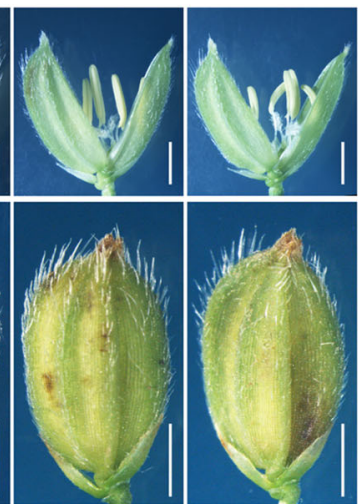

C\#1

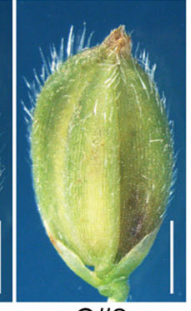

C

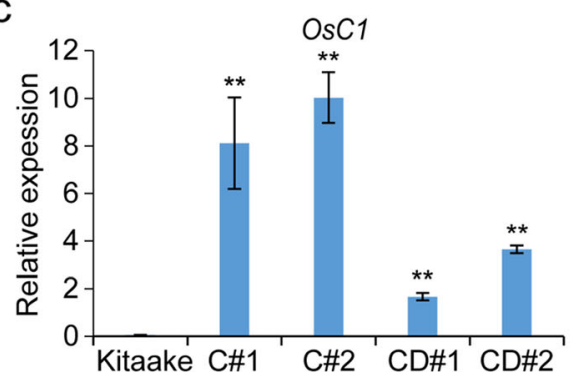

b
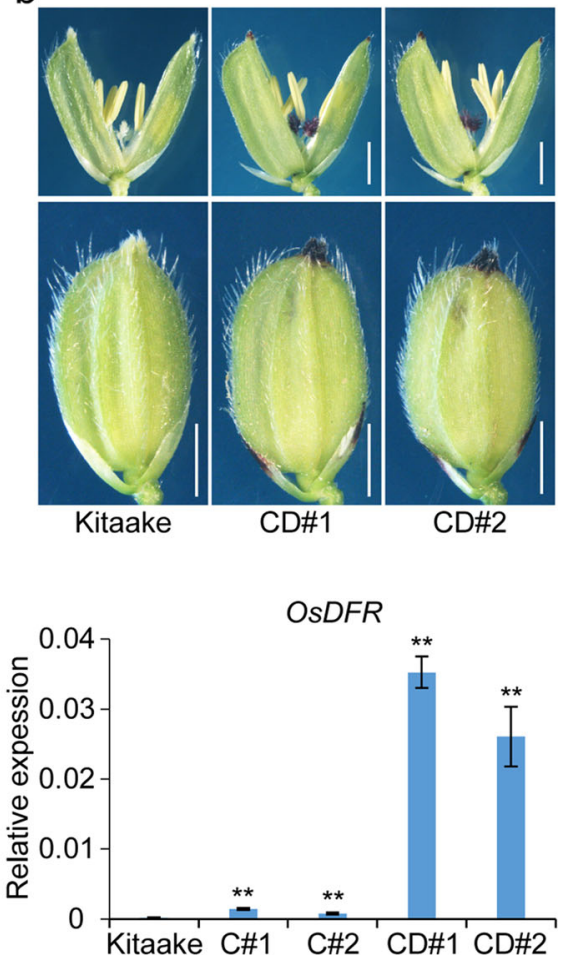

Fig. 2 Functional validation and expression analysis of OsC1 and OsDFR. a The straw-white apiculi became brown whereas stigmas remained straw-white after OsC1 was introduced into Kitaake. The top, spikelets 1 day post heading; the bottom, spikelets 14 days post heading. Bars, 2 mm. b Both apiculus and stigma became purple when both OsC1 and OsDFR were transformed into Kitaake. The top, spikelets 1 day post heading; the bottom, spikelets 14 days post heading. Bars, 2 mm. c Expression levels of OsC1 and OsDFR in Kitaake and complementation plants. C\#1, C\#2, two independent OsC1-complemented lines. CD\#1, CD\#2, two independent complemented lines with OSC1 and OsDFR. Data are means \pm SD of three biological replicates (Student's t-test: **P $<0.01$ ) 

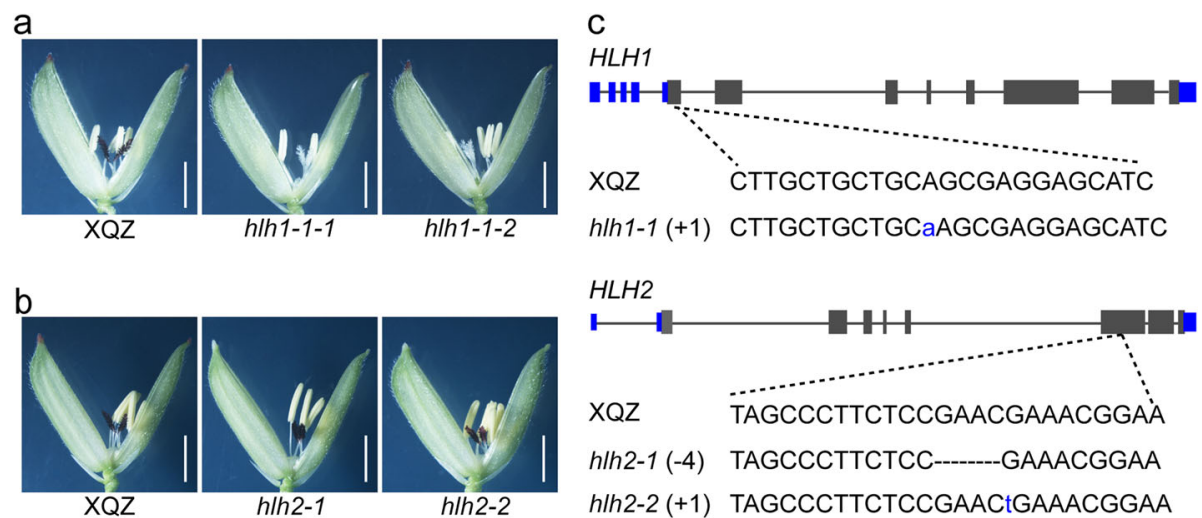

Fig. 3 Phenotypes of HLH1- and HLH2-knockout lines in XQZ background. a Knockout of HLH1 caused loss of purple stigma in XQZ. Bar, 2 mm $\mathbf{b}$ Apiculus color changed from purple to straw-white in HLH2 knockout lines of XQZ. Bars, 2 mm. c Sequence alignment between XQZ and HLH1and HLH2-knockout lines. + and - signs indicate base insertions (in blue) and deletion (by dashes), respectively, relative to XQZ. Gray boxes denote coding sequences of $H L H 1$ and $H L H 2$, and blue boxes are the untranslated regions. h/h1-1, HLH1-transgenic knockout line. hlh1-1-1and h/h1-1-2, two individuals of h/h1-1-transgenic line. h/h2-1 and h/h2-2, independent HLH2-transgenic knockout lines

TFs (Table S2) caused no obvious color variation in XQZ and LTH plants. The hlh1-1 mutant had a $1 \mathrm{bp}$ insertion at the site 57, and the hlh1-2 and hlh1-3 mutants had 4 or $6 \mathrm{bp}$ deletions in the fifth extron of LOC_Os04g47080 $(H L H 1)$, respectively, all of which caused loss of $H L H 1$ function (Fig. 3c, Fig. S6c), resulting in straw-white stigmas but purple apiculi (Fig. 3a, Fig. S6a). The hlh2-1 and hlh22 mutants contained a $4 \mathrm{bp}$ and $1 \mathrm{bp}$ insertions at sites 663 and 667, and the hlh2-3 and hlh2-4 mutants had 2 bp and $1 \mathrm{bp}$ insertions in the third extron of LOC_Os04g47040 $(H L H 2)$, respectively, all of which caused premature termination of translation (Fig. 3c, Fig. S6c), resulting in strawwhite apiculi but purple stigmas (Fig. 3b, Fig. S6b). We concluded that $H L H 1$ and $H L H 2$ are tissues-specific genes responsible for anthocyanin biosynthesis and accumulation in stigmas and apiculi of XQZ, respectively. We tentatively named them as OsPs (purple stigma) and $\mathrm{OsPa}$ (purple apiculus).

$\mathrm{OsPa}$ and $\mathrm{OsPs}$ both encode bHLH-type TFs containing a basic region in the $\mathrm{N}$-terminal related to binding of cis-regulatory DNA elements and a hydrophobic HLH region in the $\mathrm{C}$-terminal that functions as a homo-/hetero-dimerization domain (Fig. S7b, c). Interestingly, these two genes and another tissue-specific gene $S 1$ (i.e. OsB2), which determines anthocyanin accumulation in rice hulls, were located in a $65 \mathrm{~kb}$ cluster on chromosome 4 (Fig. S7d), sharing 43.81 to $57.07 \%$ identity in full-length amino acid sequence and highly conserved basic regions (73.51 to $84.24 \%$ identity) and HLH domains (84.00 to $88.00 \%$ identity) (Fig. S8; Table S3).

\section{Expression Patterns of OsC1, OsPa, OsPs and OsDFR} qRT-PCR analyses revealed that OsC1, OsPa, OsPs, and $O s D F R$ were all expressed in a range of tissues, including seedling roots, stems, leaf blades, leaf sheathes, hulls, apiculi and stigmas in XQZ plants (Fig. 4a-d), indicating that all these four genes were constitutively expressed at different developmental stages and in all tissues. However, the strongest expression of OsC1 and OsDFR was detected in the respective purple-colored tissues, i.e. stigmas and apiculi, whereas there was relatively weak expression in other tissues (Fig. 4a, b). Strikingly, $\mathrm{OsPa}$ was the most strongly expressed in apiculi with expression levels 9- and 144-fold higher than that in leaves (the 2nd strongest expression tissue) and stigmas, respectively (Fig. 4c); and OsPs was most strongly expressed in stigmas with expression levels 28- and 588fold higher than that in hulls (the 2nd strongest expression tissue) and apiculi (Fig. 4d). OsPa and OsPs showed similar expressional patterns in both the pigmented LTH and the non-pigmented Kitaake and Nipponbare (Fig. 4c, d). These data indicated that the preferential expression of these genes in different tissues may underlie their tissue-specific functionality.

\section{Subcellular Localization of OsC1, OsPa, OsPs and OsDFR} To determine the subcellular localizations of $\mathrm{OsC1}$, OsPa, OsPs and OsDFR, their full-length coding sequences (CDSs) were amplified and fused to the $\mathrm{N}$ terminus of green fluorescent protein (GFP) in a PAN580 vector. When transiently expressed in rice protoplasts, the OsC1-, OsPa-, OsPs-GFP signals were co-localized with the nuclear marker D53-mCherry, indicating that these three proteins were localized in the nucleus (Fig. S9), and matched their functioning in the nucleus. The OsDFR-GFP signal was co-localized with the nucleus and cytoplasm marker mCherry, indicating that the OsDFR protein is localized in the nucleus and cytoplasm (Fig. S9). 

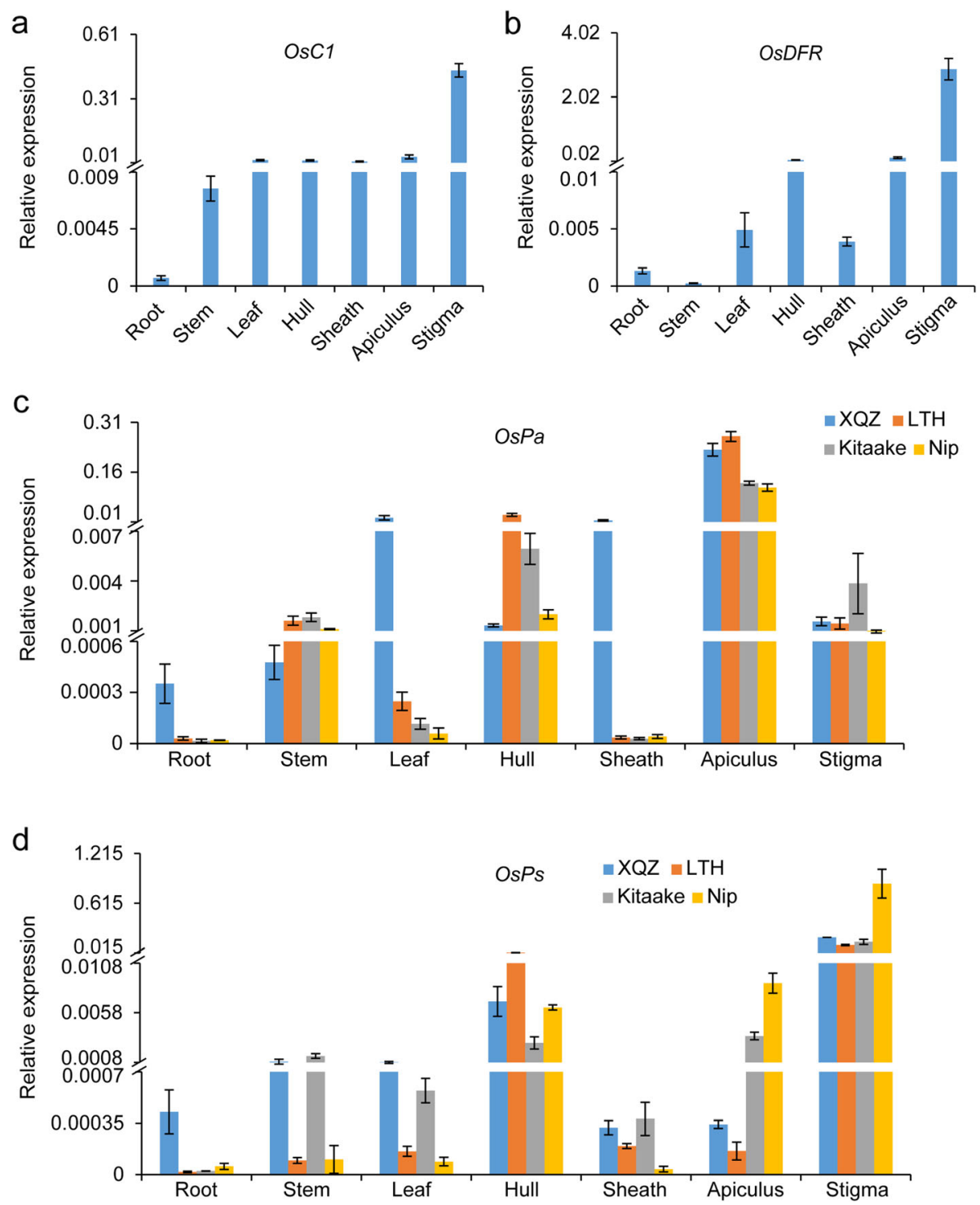

Fig. 4 Expression patterns of OsC1, OsDFR, OsPa and OsPs. Expression analysis of OsC1(a), OsDFR (b) in XQZ. Expression patterns of OsPa (c) and OsPs (d) in XQZ, LTH, Kitaake and Nipponbare (Nip). The samples were collected from roots of 10-day-old seedlings, stems, flag leaves, hulls, leaf sheaths, apiculi, and stigmas at heading, respectively. Data are presented as means \pm SD $(n=3)$

\section{Interactions between OsC1 and OsPa or OsPs}

Previous studies indicated MYB-type TFs interacted with tissue-specific genes to regulate expression of structural genes causing anthocyanin biosynthesis (Goff et al. 1990, 1992; Sun et al. 2018). We thus firstly used yeast two-hybrid assays to test the interactions of OsC1 with OsPa and OsPs. As expected, OsC1 indeed interacted with OsPa or OsPs (Fig. 5a). To further verify these interactions, we performed luciferase complementation imaging (LCI) assays and the bimolecular fluorescence complementation (BiFC) assays in Nicotiana benthamiana ( $N$. benthamiana) leaves. For these assays, OsC1 was fused to N-terminal of LUC (n-LUC) to produce $\mathrm{nLUC}-\mathrm{C} 1$, and OsPa and OsPs were fused to cLUC to generate cLUC-Pa and CLUC-Ps. As shown in Fig. 5b,
OsC1 interacted strongly with OsPa or OsPs, displaying strong luminescence signals whereas the negative controls lacked luminescence signals. In the $\mathrm{BiFC}$ assays, strong fluorescence signals appeared in the cell nuclei when OsC1 was transiently co-expressed with OsPa or OsPs, but no signals appeared in cells co-expressing $\mathrm{Yn}$ and $\mathrm{Yc}-\mathrm{OsC1}, \mathrm{Yc}$ and $\mathrm{Yn}-\mathrm{Pa}$, Yc and Yn-Ps, or Yn and Yc as controls (Fig. $5 \mathrm{c})$. These results confirmed that $\mathrm{OsC1}$ could interact with OsPa or OsPs not only in yeast but also in planta.

\section{The OsC1-OsPa and OsC1-OsPs Complexes Activate Structural Gene Expression}

We next performed dual-luciferase (LUC) assays in $N$. benthamiana leaves to test the effects of OsC1, 

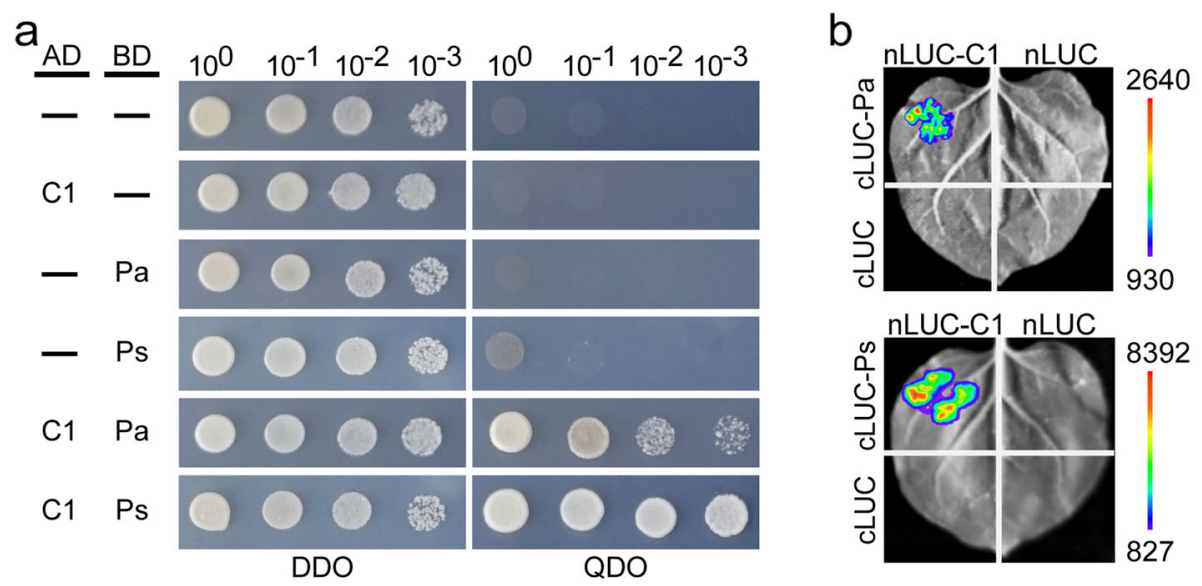

\section{C}
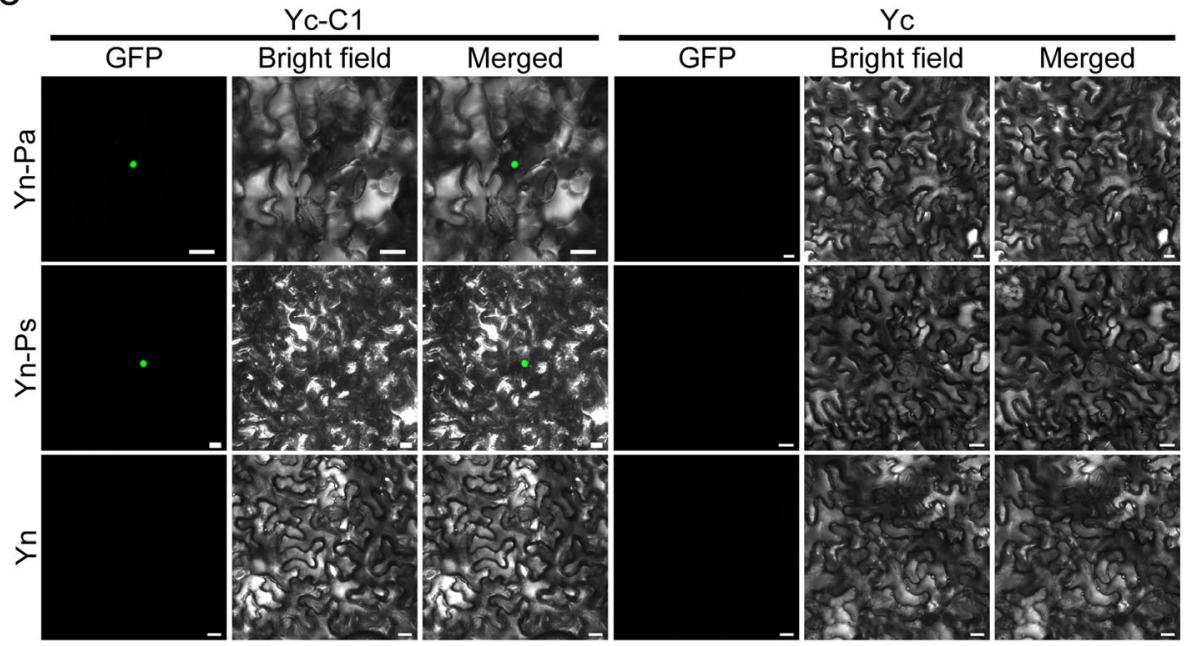

Fig. 5 OsC1 physically interacted with OsPa and OsPs. a Validation of interactions of OsC1 with OsPa and OsPs in yeast two-hybrid assays after 3 days. Transformed cells were spotted on the control (DDO, SD/-Leu/-Trp) and selective (QDO, SD/-Leu/-Trp/-His/-Ade) media. b LCl assays certified that OsC1 interacted with OsPa or OsPs. c BiFC assays for the determining interaction of OsC1 with OsPa or OsPs. The full-length OsC1 protein was fused with C-terminal YFP (Yc). Full-length OsPa and OsPs proteins were fused with N-terminal YFP (Yn), respectively

OsPa and OsPs on transcriptional expression of OsDFR. As indicated in Fig. 6a, b, OsC1, OsPa or OsPs each alone could barely activate OsDFR, however, co-expression of $\mathrm{OsC1}$ and $\mathrm{OsPa}$ or $\mathrm{OsC1}$ and OsPs significantly activated OsDFR, indicating that activation of OsDFR depended on formation of OsC1-OsPa or OsC1-OsPs complexes (Fig. 6b). The OsC1-OsPa or OsC1-OsPs complex could also initiate expression of other anthocyanin biosynthesis genes, such as CHS, CHI, F3'H, F3H and ANS (Fig. $\mathrm{S} 10)$. We postulated that OsPa or OsPs as a bHLH partner of OsC1 (R2R3-MYB) is required for activation of OsDFR and other structural genes for OsC1dependent anthocyanin biosynthesis, finally determining specific anthocyanin accumulation in apiculi or stigmas.
Functional OsPa and OsPs Potentially Pre-Exist in Almost all Natural Rice Accessions

Previous studies revealed that $O s C 1$ and OsDFR played determinant roles in evolution of the anthocyanin biosynthesis pathway (Sun et al. 2018; Zheng et al. 2019). Here, we identified tissue-specific genes, $\mathrm{OsPa}$ and $O s P s$, which also played crucial roles for purple-colored pigmentation in apiculi and stigmas, respectively. To comprehensively decipher color diversification patterns in rice, we analyzed sequence variations in the CDSs of $\mathrm{OsPa}$ and OsPs in a panel of 234 rice accessions including the sequencing reference variety Nipponbare, 175 varieties from the mini-core collection (Zhang et al. 2011) and 58 varieties carrying purple apiculi and stigmas (Table S4). 

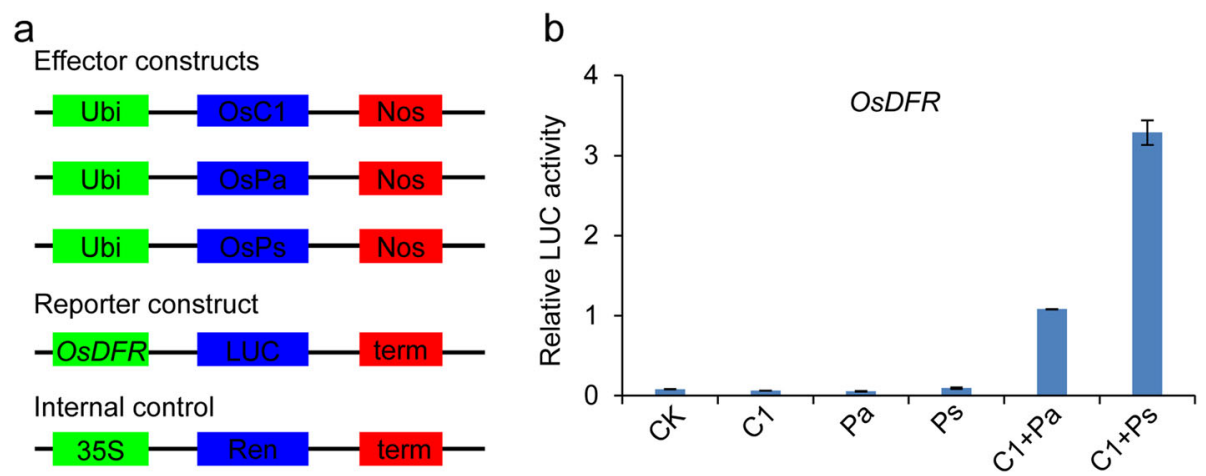

Fig. 6 Both OsC1 and OsPa or OsPs were necessary for the transcriptional expression of OsDFR promoter. a Schematic representation of the effector and reporter constructs. Full-length coding regions of OsC1, OsPa and OsPs under control of the ubiquitin promoter were used as the effectors. Firefly luciferase gene LUC driven by the OsDFR promoters and the Renilla luciferase gene Ren driven by the 355 promoter were used as the reporter and internal control, respectively. $\mathbf{b}$ Transient dual-luciferase assays were performed in Nicotiana benthamiana leaves to investigate the effects of OsC1, OsPa and OsPs on the transcriptional expression of OsDFR. Relative LUC activity was measured by the ratio Firefly luciferase (LUC): Renilla luciferase (REN), and data are presented as means \pm SD $(n=3)$

For $\mathrm{OsPa}$, seven natural variations including six nonsynonymous single nucleotide polymorphisms (SNPs) and one $18 \mathrm{bp}$ InDel were detected in the panel of 234 accessions (Fig. 7a; Table S4), and all were distributed in both the accessions with purple or brown apiculi and those with straw-white apiculi (Table S4), indicating that none of these variations affected the function of OsPa. Thirteen $\mathrm{OsPa}$ haplotypes were defined based on these seven variations (Fig. 7a); among them $\mathrm{Pa}$-Hap1 and $\mathrm{Pa}$-Hap 2 were prevailed at frequencies of 47.44 and $43.16 \%$, respectively, followed by Pa-Hap 6 with a frequency of $4.27 \%$. The other 10 haplotypes were quite rare with frequencies ranging from 0.43 to $0.85 \%$. The three prevalent haplotypes $(95.28 \%)$ were distributed in both the accessions with purple or brown apiculi and those with straw-white apiculi (Fig. 7a; Table S4). Therefore, we deduced that functional $\mathrm{OsPa}$ allele could pre-exist in almost all the natural rice accessions.

For OsPs, a total of eleven variations including eight nonsynonymous SNPs and three InDels were detected, and among them only the InDell variation caused premature termination of translation leading to loss of function of OsPs, and existed in only four accessions with straw-white stigmas (Aijiaonante, Jinzhinuo, Sankecun and Haoxiang). Due to their distribution in both pigmented and non-pigmented accessions it was clear that the other 10 variations did not affect stigma color (Fig. 7b; Table S4). Eighteen OsPs haplotypes were defined based on these 11 variations (Fig. 7b; Table S4). Among them, Ps-Hap3 was the most prevalent haplotype at $37.18 \%$, Ps-Hap15, Ps-Hap5, Ps-Hap18, Ps-Hap1, PsHap6 and $P s$-Hap9 were moderately prevalent haplotypes with occurrence frequencies ranging from 6.41 to $16.67 \%$, and the other 11 haplotypes were rare $(0.43$ to 1.71\%). Except Ps-Hap10, which was the haplotype carrying the Indel1 variation, all other haplotypes except the five extremely rare ones, Ps-Hap11-14 and PsHap16 (accounting for 2.1\%), included accessions with pigmented stigmas (Fig. 7b; Table S4). We again deduced that the functional OsPs allele could pre-exist in the majority of rice accessions.

In order to investigate the roles of promoter sequence variations in the specific expression and regulation of the transcription factors, we sequenced the $2.0 \mathrm{~kb}$ promoter regions of $O s P a$ and $O s P s$ and analyzed their sequence variations in the panel of 234 rice accessions (Table S5). As a result, 37 variations including16 for the $O s P a$ promoter region (13 types) and 21 for the OsPs promoter region (11 types) were detected, and all were distributed in both pigmented and non-pigmented accessions (Fig. S11a, b; Table S5). The result indicated that none of these variations caused functional differences of $\mathrm{OsPa}$ and OsPs between pigmented and nonpigmented rice accessions, and thus there might not be sequence-specific expression elements in the promoter regions of $\mathrm{OsPa}$ and $\mathrm{OsPs}$ that regulated their specific expression.

\section{Correlation of Haplotype Combinations of OsC1, OsDFR, $\mathrm{OsPa}$ and $\mathrm{OsPs}$ with Apiculus and Stigma Color Variations in Rice Accessions}

Phenotypic analyses of the 176 rice varieties showed that $23.29 \%$ (41 accessions) possessed purple apiculi and purple stigmas, 13.64\% (24) possessed brown/red apiculi but straw-white stigmas, and 63.07\% (111 accessions) had straw-white apiculi and straw-white stigmas (Tables S4, S5). In order to investigate the correlation between the color phenotypes and haplotype combinations of $\mathrm{OsC1}$, $O s D F R, O s P a$ and $O s P s$, we further analyzed sequence variations of $O s C 1$ and $O s D F R$ in these accessions by 


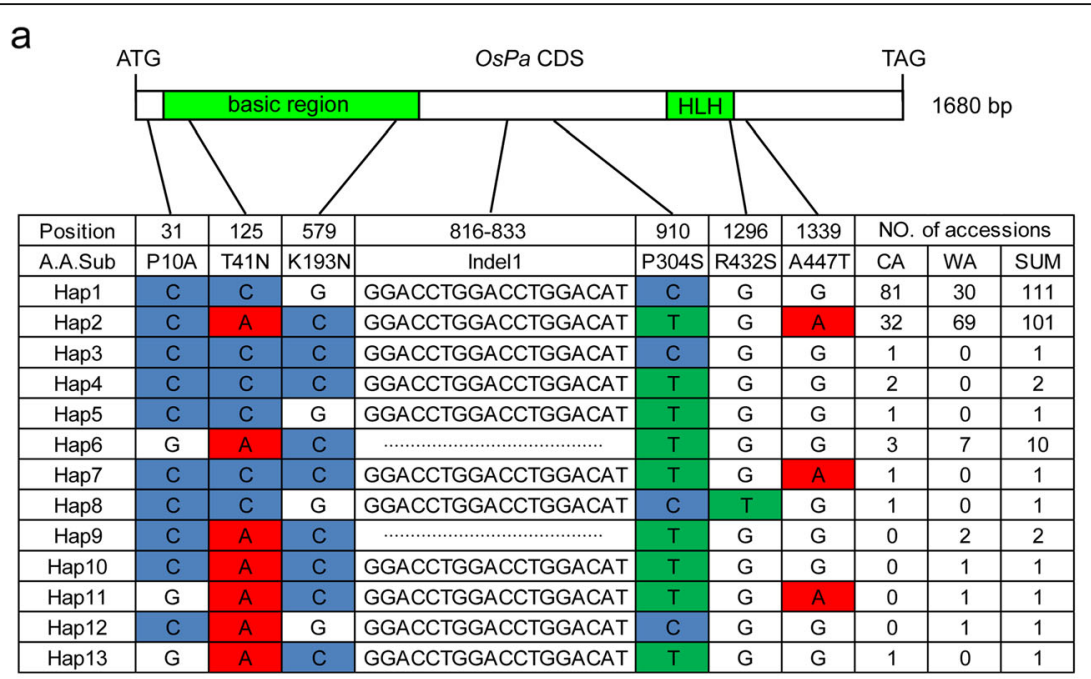

b

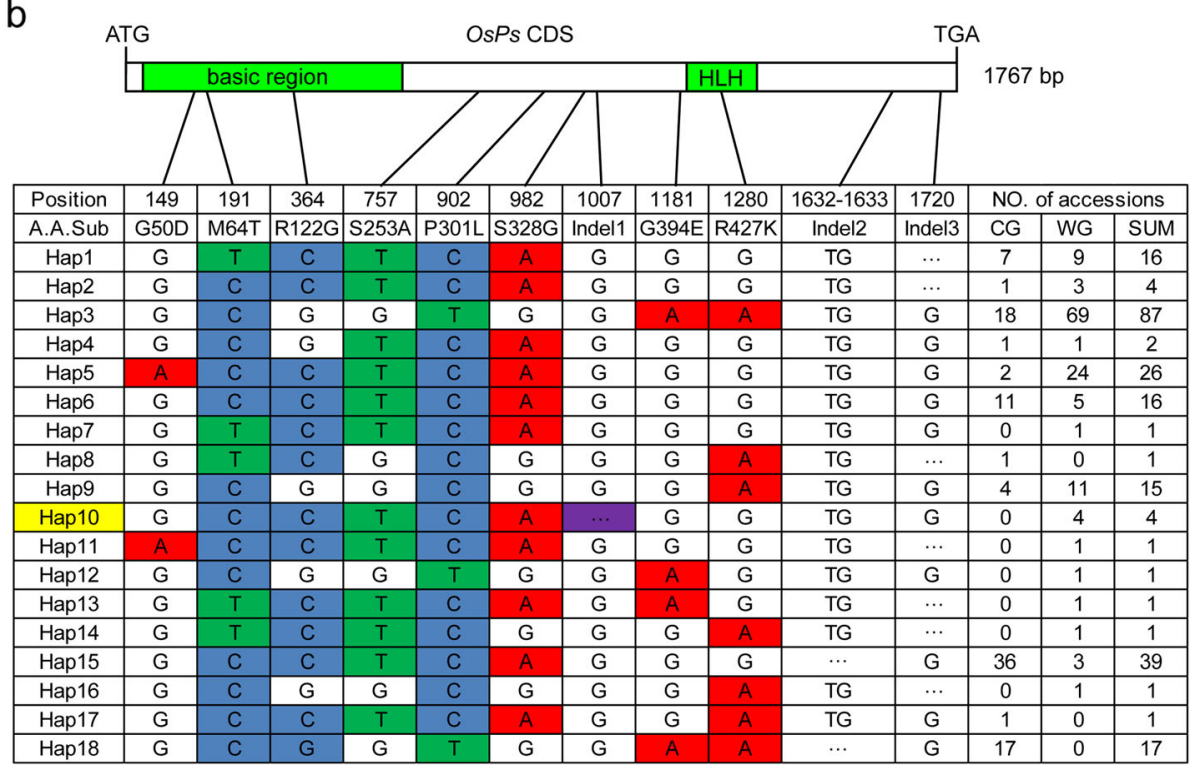

Fig. 7 Haplotype analysis of OsPa and OsPs in a nature rice panel. Haplotype analysis of OsPa (a) and OsPs (b) in 234 rice accessions. A.A.Sub are amino acid substitutions. CA, colored apiculi; WA, straw-white apiculi; CG, colored stigmas; WG, straw-white stigmas. Deletion and insertion sites are indicated by dashed lines. Haplotype analysis of OsPa and OsPs was carried out with reference to the sequences of Nipponbare (Hap1). A non-functional haplotype is indicated in yellow. Polymorphic nucleotides causing loss-of-function are in purple. The number of accessions for each haplotype is shown in the right column

sequencing their CDSs. For OsC1, two nonsynonymous SNPs, three InDels and a 45 bp substitution (Sub1) were detected, and three functional haplotypes (C1-Hap2-4) and four non-functional haplotypes (C1-Hap1 and C1Hap5-7) were identified (Fig. 8a; Table S6). For OsDFR, seven nonsynonymous SNPs were detected, and seven functional haplotypes (DFR-Hap2-4, DFR-Hap8 and $D F R$-Hap11-13) and six non-functional haplotypes (DFR-Hap1, DFR-Hap5-7 and DFR-Hap9-10) were identified (Fig. 8b; Table S6).

The 176 accessions were genotypically classified into six groups by functional or non-functional alleles of
OsC1, OsDFR, OsPa and OsPs. As expected, all the 41 accessions in Group I had purple apiculi and purple stigmas, the two in Group II had purple apiculi but strawwhite stigmas, all 22 in Group III had brown/red apiculi but straw-white stigmas, whereas accessions in Groups IV, V and VI had no pigment in both apiculi and stigmas (Tables S7, S8). These data corroborated the results of the genetic dissection of purple apiculi and stigmas (Figs. $\mathrm{S} 3, \mathrm{~S} 4)$ and those of the functional validation of $\mathrm{OsC1}$, OsDFR, OsPa and OsPs (Figs. 2, 3, Fig. S6). We thus concluded: 1) in the presence of $\mathrm{OsPa}$, apiculi displayed purple color when both $O s C 1$ and OsDFR were present, 

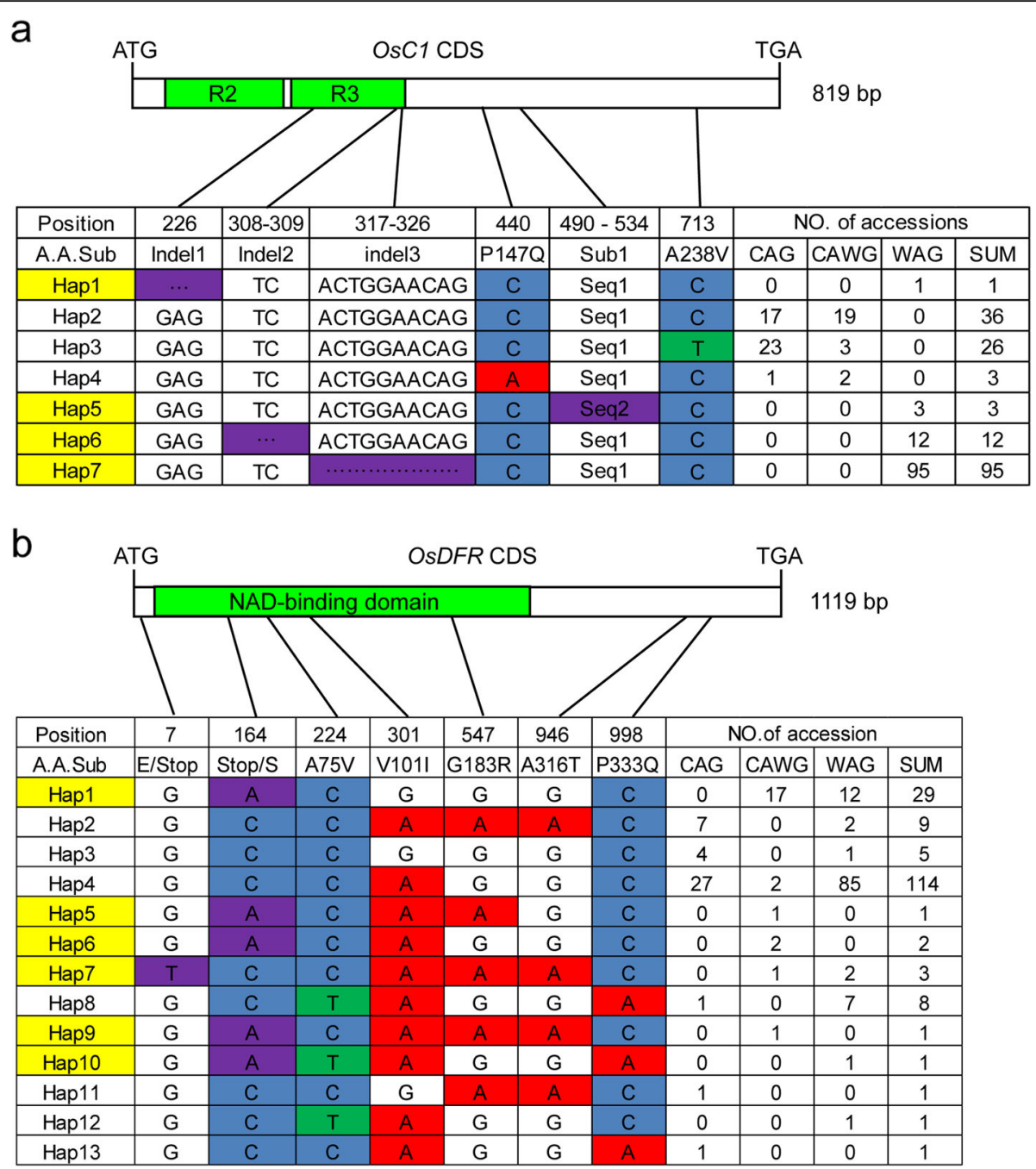

Fig. 8 Haplotype analysis of OSC1 and OSDFR in a panel of 176 rice accessions. Haplotype analysis of OsC1 (a) and OsDFR (b) in the mini core collection. A.A.Sub, indicates amino acid substitution. CAG, colored apiculi and colored stigmas; CAWG, colored apiculi and straw-white stigma; WAG, straw-white apiculi and straw-white stigma. Deletion and insertion sites are indicated by dashed lines. Haplotype analyses of OsC1 and OsDFR were conducted according to the sequences of Nipponbare (Hap1). Non-functional haplotype is in yellow. Polymorphic nucleotides causing loss-of-function are in purple. The number of accessions of each haplotype is shown in the right column. Sub1, substitution of ACGGCA GCGGCGGCGGCGGCGGCGACGACGA CCACCGTGTGGGCG (Seq1) by GCAGCCAGCCT (Seq2)

and the color changed to red or brown color when $\mathrm{OsC1}$ was present but OsDFR was absent. There was no pigmentation regardless of the presence of OsDFR when $\mathrm{OsC1}$ was absent. In the absence of $\mathrm{OsPa}$, the apiculi displayed no color regardless of the presence of both $O s C 1$ and $O s D F R ; 2)$ stigmas were purple only when all three genes $O s C 1, O s D F R$ and OsPs were present, and the absence of any one resulted in no pigmentation.

\section{Discussion}

Determinant Factors for Anthocyanin Biosynthesis in Rice Apiculi and Stigmas

The $C$ - $A$ - $P$ gene system controlling anthocyanin coloration was firstly established for purple apiculi of japonica rice (Takahashi 1957; Kondo 1963). In this system, two basic complementary genes, $C$ and $A$, were assumed to be responsible for the production of anthocyanin color together with a $P$ gene that conferred color to specific organs. The genetics of purple apiculi and purple stigmas are examples of traits that until now were largely unknown.

In the present study, we first mapped and isolated complementary genes $\mathrm{OsC1}$ and OsDFR responsible for the purple coloration of apiculi and stigmas in indica $\mathrm{cv}$. XQZ (Fig. 1, Fig. S3; Table S1). Introduction of OsC1 alone with its native promoter into cv. Kitaake caused brown apiculi but straw-white stigmas in a manner similar to that reported by Zhao et al. (2016), but in 
combination with OsDFR it produced not only purple apiculi but also purple stigmas (Fig. 2, Fig. S4). We then identified the tissue-specific pigmentation genes, $\mathrm{OsPa}$ and OsPs from shortlisted candidates by means of the phylogenetic analysis of all anthocyanin biosynthesisassociated bHLH TFs in maize and rice and CRISPR/ Cas9 knockout (Fig. S5; Table S2). Knockout of OsPa in both XQZ and LTH backgrounds caused loss of purple apiculus but retention of purple stigmas, whereas knockout of OsPs produced the opposite effect (Fig. 3, Fig. S6). In addition, qRT-PCR revealed that that $\mathrm{OsPa}$ was strongly expressed in apiculi with an expression level at least 32-fold higher than that in stigmas, and on the contrary OsPs was strongly expressed in stigmas with an expression level at least 42-fold higher than that in apiculus (Fig. 4c, d). These results provided strong evidence that $\mathrm{OsPa}$ and $\mathrm{OsPs}$ acted as tissue-specific genes and participated in the pigmentation of apiculi and stigmas, respectively. Thus, purple pigmentation of each of apiculi and stigmas was indeed controlled by at least three genes, i.e. OsC1, OsDFR and OsPa or OsPs, which respectively corresponded to alleles $C, A$ and $P$ in the $C$ $A-P$ gene system.

Based on sequencing and functional allele analysis of $O s C 1, O s D F R, O s P a$ and $O s P s$, we genotyped a panel of 176 rice accessions including the sequenced reference Nipponbare and 175 accessions from the Chinese minicore collection for their apiculus and stigma pigmentation, and grouped them into six genotype groups (I-VI) based on apiculus and stigma color (Tables S7, S8). This confirmed the general applicability of the OsC1-OsDFR$O s P a$ and OsC1-OsDFR-OsPs systems to natural rice accessions regardless of subspecies for deciphering the regulatory mechanism of anthocyanin biosynthesis in apiculi and stigmas.

\section{The Regulatory Systems of Anthocyanin Biosynthesis in Rice Apiculi and Stigmas}

The OsC1-OsDFR-OsPa and the OsC1-OsDFR-OsPs gene systems are summarized in Fig. 9. The two systems

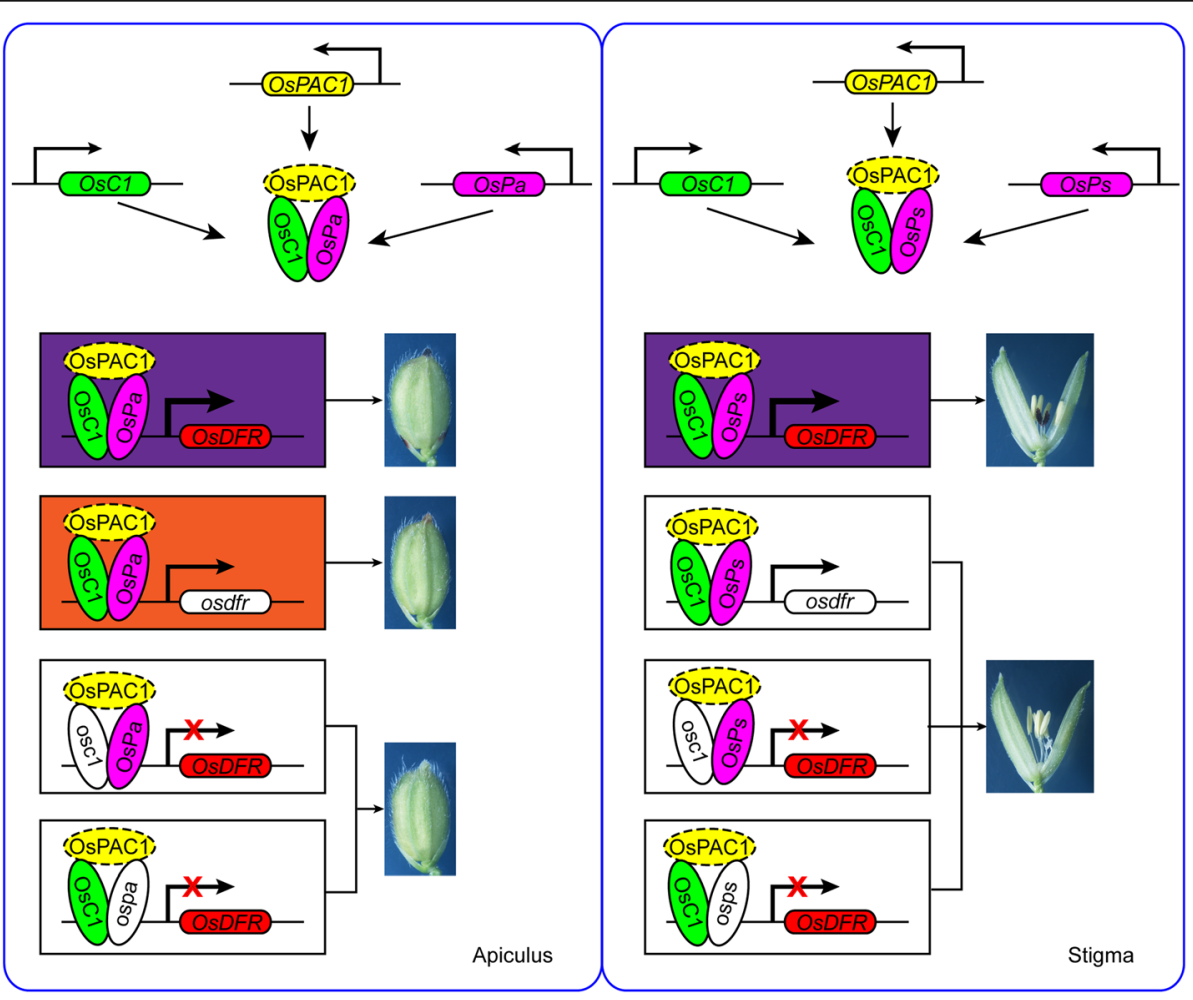

Fig. 9 Working models of apiculus and stigma coloration in rice. Both OsC1 and OsDFR had extremely high expression levels in both apiculus and stigmas, whereas OsPa and OsPs were more strongly expressed in the respective apiculi and stigmas. When all of OsC1, OsDFR, OsPa and OsPs were functional the OsC1-OsPa-OsPAC1 and OsC1-OsPs-OsPAC1 complexes activated expression of OsDFR resulting in purple apiculi and purple stigmas, respectively. When OsC1 was not functional, it affected complex formation, therefore decreasing the expression of OsDFR and leading to non-pigmented apiculi and stigmas. When OSDFR was not functional, there was no stigma color and apiculi were brown. When OsPa or OsPS were nonfunctional, both apiculi and stigmas were non-pigmented. OsC1, OsPa and OsPs represented functional proteins corresponding to the products of OsC1, OsPa and OsPs, respectively. osc1, ospa and osps represented nonfunctional products of the OsC1, OsPa and OsPs loci, respectively. OsDFR and osdfr represented functional and nonfunctional OsDFR, respectively. Solid ellipse, interaction of the protein inside with the other had been confirmed; dashed ellipse, interaction of the protein inside with the other needed to confirm 
share similar regulatory mechanisms of pigmentation as the $C-A-S$ and the OsC1-OsRb-OsDFR gene models (Sun et al. 2018; Zheng et al. 2019). That is, the R2R3-MYB TF OsC1 (i.e. C1) interacts with tissue-specific bHLH TFs (OsPa, OsPs, S1/OsB2, or OsRb) to activate and elevate the expression levels of OsDFR (i.e. A1) and other anthocyanin biosynthesis genes, causing purple color in apiculi, stigmas, hulls or leaves, respectively.

Previous studies identified several putative tissuespecific genes for anthocyanin biosynthesis including OsB1 (Ra1), OsB2, Rb1, and Rb2 (Hu et al. 1996, 2000; Sakamoto et al. 2001). Only two, OsB2 (i.e. Kala4 and $S 1$, hull-specific) and (i.e. $O s R b$, leaf-specific), had been functionally confirmed, and they encoded typical bHLHtype TFs sharing higher homology to OsPa (LOC_ Os04g47040) than to OsPs (LOC_Os04g47080) (Fig. S5; Sun et al. 2018; Zheng et al. 2019). Nevertheless, OsPa, $S 1$ and OsPs are located in the same gene cluster on chromosome 4 sharing highly conserved basic regions and HLH domains. OsPs, formerly named $R a 1$ and OsB1, had high homology along its entire length with the maize $R(L c)$ gene (Hu et al. 1996, 2000; Sakamoto et al. 2001). OsPs and its adjacent $S 1$ were earlier reported to participate in pigmentation of rice leaves (Sakamoto et al. 2001). However, the recent study of Zheng et al. (2019) suggested that both OsPs and S1 might not be connected with anthocyanin biosynthesis due to their extremely low expression levels in leaves. On the contrary, OsPs is most strongly expressed in stigmas and functions as a stigma-specific gene for anthocyanin biosynthesis (Figs. 3a, 4d, Fig. S6a), whereas S1 was still regarded as a determinant of purple hull although a 'gain-of-function' mutation in its promoter region is responsible for purple pericarp (Oikawa et al. 2015; Sun et al. 2018). To our knowledge this is the first report on $\mathrm{OsPa}$ and its function in anthocyanin biosynthesis as an apiculus-specific gene. The fact that the clustered bHLH-type TF homologs $O s P a, S 1$ and OsPs, have different tissue-specific functions could reflect the allelic constitutions at the $\mathrm{OsPa} / \mathrm{S} 1 / \mathrm{OsPs}$ regulatory loci, and may provide cues for understanding the functional diversification mechanisms of tissue-specific regulators related to anthocyanin biosynthesis in plants.

The two types of TFs in maize, a R2R3-MYB-related protein and a bHLH-containing protein, interact with each other and activate anthocyanin biosynthesis genes as a single complex (Petroni and Tonelli 2011). Our results showed that R2R3-MYB-type regulator OsC1 interacts with two bHLH-type TFs, OsPa and OsPs, respectively in determining the expression of OsDFR and some other structural genes (Figs. 5, 6, Fig. S10), leading to the tissue-specific distribution of purple pigmentation. In Arabidopsis, the WD40 repeat protein TTG1 interacts with R2R3-MYB-type TFs and bHLH-type TFs to form MYB-bHLH-WD40 complexes with roles in anthocyanin accumulation in vegetative tissues or proanthocyanin accumulation in developing seeds. The mutation of TTG1 directly leads to the appearance of yellow seed coat, and the proanthocyanin of seed coat cannot be synthesized (Xu et al. 2015). In tobacco, the transformation of anthocyanin-related R2R3-type MYB transcription factor activates the synthesis of anthocyanins that are inseparable from the expression of WD40 (Montefiori et al. 2015). PAC1, the homolog of TTG1 in maize, is required for the anthocyanin accumulation in the pericarp (Petroni and Tonelli 2011). In rice, Sun et al. (2018) assumed that OsPAC1 was not essential for anthocyanin biosynthesis because there were no functional mutations in natural rice germplasm. However, Zheng et al. (2019) assumed that OsPAC1 was required for full activation of anthocyanin biosynthesis genes by interacting with OsC1 and OsRb, and Zhu et al. (2017) found that the specific biosynthesis of anthocyanins in rice endosperm was involved in up-regulation of endogenous genes $O s W D 40$. In the present study, we found that OsPAC1 was expressed constitutively in all tissues tested (Fig. S12), and no any functional mutations in the OsPAC1 CDS occurred between the pigmented and nonpigmented rice accessions (Table S9). Therefore, we deduced that functional OsPAC1 allele could pre-exist in almost all the natural rice accessions, and be indispensable for anthocyanin biosynthesis in rice apiculi and stigmas.

In order to decipher rice color diversification patterns, we investigated the CDS variations of $O s P a$ and $O s P s$ in a panel of 234 natural rice accessions (Table S4). All seven variations in $O s P a$ and 10 of 11 variations (except InDel1) of OsPs did not affect their normal functions we deduced that all OsPa haplotypes and most OsPs haplotypes in the panel functioned normally (Tables S7, S8), perhaps explaining why these two genes were not characterized by conventional genetics and previous mapbased cloning studies (Han et al. 2006; Fan et al. 2008; Chen et al. 2010; Zhao et al. 2016). It also addresses the fact that most rice lines developed from crosses express anthocyanin color in the stigma only when the apiculus is colored (Takahashi 1964). Oka (1991) reported an upland variety Gaisen-mochi with non-pigmented apiculi and purple stigmas, and postulated a recessive inhibitor gene $i$-Ps 1 in addition to the genotype $C A p P s-1$. According to the OsC1-DFR-OsPa and OsC1-OsDFR-OsPs systems we predict that Gaisen-mochi has genotype OsC1-DFR-Ospa-OsPs, in which loss of function of OsPa is responsible for the straw-white apiculus but does not affect the coloration of stigmas. Reddy (1996) reported a leaf blade-specific dominant inhibitor of anthocyanin pigmentation $(I l b)$ in indica lines N22B and N22W, that inhibited pigmentation of the leaf blade. It was observed 
that some crosses between pigmented and nonpigmented rice lines produce non-pigmented $F_{1}$ plants, suggesting a common presence of dominant inhibitor alleles among rice cultivars (Reddy 1996). Recently, a number of repressor proteins including R3-MYB and R2R3-MYB repressors that limit expression of anthocyanin biosynthesis genes were identified in horticultural plants (Albert et al. 2014; Naing and Kim 2018; Zhang et al. 2020), strengthening the need to search for tissuespecific inhibitors of anthocyanin biosynthesis in rice.

\section{Brown Apiculi Color May Be Caused by the OsC1-OsPa Complex Rather than OsC1 Alone}

OsC1 alone was regarded as the chromogen gene responsible for the brown or red apiculi (Saitoh et al. 2004; Fan et al. 2008; Zhao et al. 2016; Sun et al. 2018). However, our CRISPR-Cas9 knockout results for $\mathrm{OsPa}$ demonstrated that $\mathrm{OsC1}$ itself does not produce color when functioning alone (Fig. 3b, Fig. S6b), but produces brown apiculi only when functioning together with $\mathrm{OsPa}$ (Figs. 6, 7, Fig. S10). This indicates that $\mathrm{OsC1}$ might not be a chromogen gene for anthocyanin, but could work as a switch in controlling color production as previously reported (Zhao et al. 2016; Sun et al. 2018).

The reasons why the OsC1-complementation and/or transgenic overexpression lines in Kitaake and Nipponbare backgrounds produced brown or red color only in the apiculi in previous studies (Zhao et al. 2016; Sun et al. 2018) could be that both possessed the genotype Osc1-Osdfr-OsPa-OsPs (Figs. 1, 2; Table S7). When $\mathrm{OsC1}$ alone was introduced into $\mathrm{HC} 1$ with genotype of Osc1-S1-osdf, the hull color of all transgenic lines was brown (Sun et al. 2018), indicating that the OsC1-S1 complex rather than $\mathrm{OsC1}$ alone caused brown hulls. The brown color of rice hulls involves accumulation of the main products as flavonols and flavanones in the absence of a functional OsDFR (Sun et al. 2018). Thus, we speculate that brown apiculi could have a similar control mechanism as brown hulls. Further work including identification of the catalytic enzyme targeted by the OsC1-OsPa complex and verification of the interaction between this enzyme and the OsC1-OsPa complex is needed to determine the regulatory systems of flavonol and/or flavanone biosynthesis in brown apiculi.

\section{Conclusion}

In the present study, we identified four determinant factors for purple apiculi and stigmas from indica cultivar Xieqingzao and dissected their regulatory mechanisms by genetic and molecular approaches. Among these determinants, $\mathrm{OsC1}$ encodes a R2R3-MYB transcriptional factor, $O s D F R$ encodes a dihydroflavonol 4-reductase, and $\mathrm{OsPa}$ and $\mathrm{OsPs}$ encode bHLH transcription factors that function as apiculus- and stigma-specific regulator, respectively. OsC1 specifically interacts with $\mathrm{OsPa}$ or OsPs to activate OsDFR and other anthocyanin biosynthesis genes, resulting in purple-colored apiculi or stigmas. OsC1 does not produce color when functioning alone, but produces brown apiculi only when functioning together with $\mathrm{OsPa}$, indicating that $\mathrm{OsCl}$ itself might not be a chromogen gene for anthocyanin. Genotyping and phenotyping of 176 rice accessions validated the general applicability of the OsC1-OsDFR-OsPa and OsC1-OsDFR-OsPs models to natural rice accessions regardless of subspecies. Our findings disclosed the biological functions of $O s C 1, O s P a$ and $O s P s$, and shed light on the specific regulatory systems of anthocyanin biosynthesis in apiculi and stigmas, a further step in understanding the regulatory network of anthocyanin biosynthesis in rice.

\section{Materials and Methods}

Plant Materials and Growth Conditions

Indica cv. XQZ (female parent) with purple apiculi and purple stigmas and japonica cv. Kitaake (male parent) with straw-white apiculi and stigmas were crossed to develop $F_{1}$ and $F_{2}$ populations for genetic analysis and gene mapping. LTH with purple apiculus and stigma was used for knockout analysis of tissues-specific genes. A panel of 234 rice accessions, including Nipponbare, 175 varieties from the mini-core collection and 58 varieties carrying purple apiculi and stigmas were used for haplotype analysis of OsC1, OsDFR, OsPa and OsPs (Table S4). All seeds were seeded in an incubator and 30-day-old seedings were transplanted and grown in a paddy field at the Institute of Crop Science Shunyi Experimental Station in Beijing during April-October.

\section{Extraction and Quantification of Anthocyanin}

Fifty milligrams of apiculi and stigmas respectively were isolated from rice florets 1 day post heading. Total anthocyanins from apiculi and stigmas were extracted and quantified as previously reported (Mehrtens et al. 2005). All samples were measured with three biological replicates.

\section{Positional Cloning of OsC1 and OsDFR}

To map the target genes determining apiculus and stigma color, 212 InDel markers covering all the 12 chromosomes (Wang et al. 2017) were used for initial screening for polymorphisms between XQZ and Kitaake. Molecular markers used for fine mapping were designed based on the nucleotide polymorphisms between japonica cv. Nipponbare and indica cv. 9311. Annotation of predicted ORFs (open reading frames) in the candidate region was based on the rice genome annotation project database (http://rice.plantbiology.msu.edu/cgi-bin/ gbrowse/rice/). 


\section{Vector Construction and Rice Transformation}

A $4.8 \mathrm{~kb}$ genomic fragment of $\mathrm{OsCl}$ consisting of a 2.5 $\mathrm{kb}$ promoter region, the entire $\mathrm{OsC1}$ coding region, and a $1.0 \mathrm{~kb}$ downstream region was amplified from $\mathrm{XQZ}$ with the primer pairs 1305-C-F/1305-C-R and inserted the SalI and AflII restriction sites of the pCAMBIA1305.1 vector to generate transformation plasmid proOsC1:OsC1 which was then introduced into calli of Kitaake via Agrobacterium-mediated transformation. A $5.9 \mathrm{~kb}$ DNA fragment of OsDFR covering the entire coding region plus $2390 \mathrm{bp}$ upstream and $1921 \mathrm{bp}$ downstream regions was amplified using primers 2300-DFRF/R and inserted EcoRI/SmalI restriction sites of the binary vector pCAMBIA2300 to generate a fused proDFR: $D F R$ construct which was introduced into calli of Kitaake and transgenic plants that already carried the the above $4.8 \mathrm{~kb}$ genomic fragment of $\mathrm{OsCl}$.

To knock out six tissue-specific genes (Table S2) 18 or 20 bp gene-specific spacer sequences were cloned into the vector sgRNA-Cas9 (Duan et al. 2019) and subsequently introduced into the calli of XQZ and LTH via Agrobacterium-mediated transformation.

\section{Structure Analysis and Multiple Sequence Alignment}

The functional domains of OsC1, OsPa and OsPs were analyzed using the Simple Modular Architecture Research Tools (SMART) program (http://smart.emblheidelberg.de/smart/set_mode.cgi?NORMAL=1). Multiple sequence alignment was conducted by DNAMAN. The Gramene database (http://www.gramene.org/) were accessed to search for rice homologs of maize $R(B$ peru).

\section{Subcellular Localizations of OsC1, OsDFR, OsPa and OsPs} For subcellular localization, coding sequences (CDSs) of $O s C 1$, OsDFR, OsPa and OsPs without stop codons were amplified and recombined into N-terminal of GFP in the pAN580 vector under the control of the CaMV 35S promoter. Fusion expression vectors were co-transformed into rice protoplasts with the nuclear marker D53mCherry or empty-mCherry. Laser confocal scanning microscope (ZEISS Microsystems LSM 700) was used to detect fluorescence signals.

\section{RNA Extraction and qRT-PCR Analysis}

RNA was extracted from young roots of 10-day-old seedlings, stems, flag leaves, hulls, leaf sheaths, apiculi and stigmas at heading using a ZR Plant RNA MiniPrep Kit (Zymo Research) following the manufacturer's instructions. Reverse transcription and qRT-PCR were conducted as described previously (Wang et al. 2017) The rice $\mathrm{Ubi-}$ quitin gene (LOC_OsO3g13170) was used as an endogenous control, and the $2^{-\Delta \Delta C T}$ method was used to evaluate relative levels of gene expression (Ma et al. 2019). The primer used for qRT-PCR was designed with GenScript (https://www.genscript.com/ssl-bin/app/primer).

\section{Yeast Two-Hybrid Assays}

The full length of $\mathrm{OsCl}$ coding region was cloned and inserted into EcoRI/XhoI restriction sites of prey vector pGADT7 and the entire coding regions of $\mathrm{OsPa}$ and OsPs were amplified and recombined into a vector pGBKT7 bait. Various combinations of prey and bait vectors were co-transformed into yeast strain AH109 (Clontech). After 3 days of growth on SD-Trp/-Leu plates at $30^{\circ} \mathrm{C}$, the interactions between baits and preys were determined on selective media (SD-Leu/-Trp/-His/-Ade) at $30^{\circ} \mathrm{C}$. All assays were performed with three repeats.

\section{LCl Assays}

Protocols used for LCI assays were as published (Chen et al. 2008). The CDS of OsC1 without stop codon was fused into BamHI/SalI restriction sites of pCAMBIA1300-nLUC to generate the recombined construct nLUC-C1 under the control of the CaMV35S promoter. The CDSs of OsPa and OsPs were amplified and cloned into the KpnI/SalI restriction sites of pCAMBIA1300-cLUC to generate the fused constructs cLUC-Pa and cLUC-Ps with the CaMV35S promoter. The recombinants were then introduced into Agrobacterium tumefaciens strain EHA105. The combined nLUC and cLUC constructs or corresponding empty vectors were co-infiltrated into in $N$. benthamiana leaves. The transient dual-luciferase image was captured using a low-lightcooled CCD imaging apparatus (Night SHADE LB 985 [Berthold] with Indigo software) 48-72 h after infiltration.

\section{BiFC Assays}

For the BiFC assays, full-length of OsPa and OsPs proteins were fused with N-terminal YFP (nYFP) to generate $\mathrm{Yn}-\mathrm{Pa}$ and $\mathrm{Yn}-\mathrm{Ps}$ constructs, respectively. OsC1 was fused with C-terminal YFP (cYFP) to generate Yc-OsC1 construct. The combined constructs $\mathrm{Yc}-\mathrm{OsC1} / \mathrm{Yn}-\mathrm{Pa}$, Yc-OsC1/Yn-Ps, Yc-OsC1/Yn were transiently coexpressed in N. benthamiana leaves via Agrobacteriummediated transformation. Fluorescence was observed by confocal microscopy (ZEISS Microsystems LSM 700) 2 days after transformation.

\section{Transactivation Activity Assays}

An approximately $2.5 \mathrm{~kb}$ promoter region of OsDFR was amplified from XQZ genomic DNA and cloned into the pGreenII 0800-LUC vector to generate a $D F R_{\text {pro- }}-L U C$ reporter construct. The full-length CDSs of OsC1, OsPa and OsPs were amplified and recombined into the BamHI/KpnI restriction sites in the pCUbi1390 vector to generate effector constructs. The combined reporter and effector plasmids were introduced into 
Agrobacterium strains EHA105, and then transiently coexpressed in N. benthamiana leaves as described previously (Waadt and Kudla 2008). The luciferase gene from Renilla reniformis (Ren) under control of the CaMV35S promoter was used as the internal control. LUC activity was calculated with a Promega Kit (E2920) following the manufacturer's instructions 48-72 h after transformation and the relative LUC activity was represented by the ratio of LUC/Ren.

\section{Primers}

All the primers used in the study are listed in Supplementary Table S10.

\section{Supplementary Information}

The online version contains supplementary material available at https://doi. org/10.1186/s12284-021-00480-1.

Additional file 1: Fig. S1. Color phenotypes of indica cultivar XQZ. (a) Purple stigma development of XQZ 2, 1 and 0 days before heading (DBH). Bars, $2 \mathrm{~mm}$. (b) Purple apiculi development of XQZ 2 and 0 days before heading $(\mathrm{DBH})$, and 7 days after heading $(\mathrm{DAH})$. Bars, $1 \mathrm{~mm}$.

Additional file 2: Fig. S2. Relative anthocyanin contents of apiculi and stigmas one day post heading in XQZ and Kitaake. Data are means \pm SD of three biological replicates (Student's $t$-test: ${ }^{* *} P<0.01$ ).

Additional file 3: Fig. S3. Inheritance of apiculi and stigma coloration. (a) Kitaake with straw-white apiculi and stigma was crossed with XQZ with purple apiculi and stigma. (b) $F_{1}$ individuals exhibited purple apiculi and stigmas similar to XQZ. (C) The $F_{2}$ population segregated into three phenotypes fitting a 9:3:4 ratio (apiculi and stigmas both purple; apiculi brown, stigmas straw-white; apiculi and stigmas both straw-white).

Additional file 4: Fig. S4. Apiculus color development in Kitaake, XQZ and transgenic complemented lines. Apiculus color development of (a) Kitaake, (b) OsC1-transgenic complemented lines, (c) OsC1 and OsDFRtransgenic complemented lines, and (d) XQZ. St1 to St6 are $-1,1,7,14$, 21 , and 26 days post heading.

Additional file 5: Fig. S5. Phylogenetic tree of all rice bHLH transcriptional factors (TFs) and known maize bHLH TFs associated with anthocyanin biosynthesis. The genes in blue denote known maize bHLH TFs associated with anthocyanin biosynthesis. The genes highlighted in red represented are the rice bHLH TFs closest to the known maize bHLH TFs. The tree was constructed using MEGA 5.2 and bootstrapped with 1000 replicates.

Additional file 6: Fig. S6. Phenotypes of HLH1 and HLH2 knockout mutants in the LTH background. (a) the stigma color changed from purple to straw-white in $h / h 1$ mutants. Bar, $1 \mathrm{~mm}$. (b) Purple apiculus color is lost in h/h2 mutants. Bar, $1 \mathrm{~mm}$. (c) Sequencing of the CRISPR/ Cas9-targeted sites of HLH1- and HLH2- knockout lines. Plus (+) and minus (-) indicate base insertions (in blue) and deletions (by hyphen), respectively, relative to LTH. Gray boxes denote coding sequences of HLH1 and $H L H 2$, and blue boxes are the untranslated regions. h/h $1-2$ and $h / h 1-3$ are independent HLH1-transgenic knockout lines. h/h $1-2$ has a six-base deletion at position 52, causing a two-amino acids deletion. h/h1-3 has a four-base deletion at position 54, causing a premature termination of translation. h/h2-3 and h/h2-4 are independent HLH2-transgenic knockout lines. h/h2-3 has a two-base deletion, and h/h2-4 has a single base insertion, both causing premature termination of translation.

Additional file 7: Fig. S7. Protein structures of OsC1, OsPa, OsPs and physical distance analysis of among three bHLH transcriptional factors (TFs). (a-c) Protein structures of OsC1, OsPa and OsPs. Numbers above the diagrams indicate residue positions. R2R3 repeats, basic regions and hydrophobic $\mathrm{HLH}$ regions are labelled with green boxes. (d) Physical distances separating three bHLH TFs on chromosome 4 indicated above the genes.

Additional file 8: Fig. S8. Amino acid sequence alignments of OsPa, OsPs and S1 determined using DNAMAN. Consensus amino acids are shown at the bottom with lowercase letters; black indicates 100\% identify; green indicates > 50\% identify; and yellow indicates > 33\% identify. Red and blue lines indicate the basic region and HLH domain, respectively.

Additional file 9: Fig. S9. Subcellular localization of OsC1, OsPa, OsPs and OsDFR in rice protoplasts. The OsC1-, OsPa-, OsPs-GFP fusion protein were transiently co-expressed with nuclear marker D53-mCherry in rice protoplasts. OsDFR-GFP fusion protein was co-expressed with emptymCherry in rice protoplasts. Left to right: images of GFP (green), mCherry (red), protoplast, and merged GFP and mCherry.

Additional file 10: Fig. S10. Transient activation assays of OsC1, OsPa and OsPs on the promoters of five structural genes. (a) Schematic representation of the effector and reporter constructs. Full-length coding regions of OsC1, OsPa and OsPs under control of the ubiquitin promoter were used as the effectors. The Firefly luciferase gene LUC driven by the five structural genes promoters and the Renilla luciferase gene Ren driven by the 355 promoter were used as reporter and internal control, respectively. (b-f) Transient dual-luciferase assays were performed in Nicotiana benthamiana leaves to investigate the effects of OsC1, OsPa and OsPs on the transcriptional expression of structural genes. Relative LUC activity was measured by Firefly luciferase (LUC): Renilla luciferase (REN) ratio and data are presented as mean \pm SD $(n=3)$.

Additional file 11: Fig. S11. Sequencing of $2.0 \mathrm{~kb}$ promoter regions of OsPa and OsPs in a panel of 234 rice accessions. Promoter sequence variations of OsPa (a) and OsPs (b) in the 234 rice accessions. B. sub are base substitutions. CA, colored apiculi; WA, straw-white apiculi; CG, colored stigmas; WG, straw-white stigmas. Deletion and insertion sites are indicated by dashed lines. Promoter sequence analysis of OsPa and OsPs was carried out with reference to the sequences of Nipponbare (T1). Seq1, CTAAAAT; Seq2, ACGACACT. The numbers at the top represented the base variation positions from the start codon.

Additional file 12: Fig. S12. Expression analysis of OSPAC1 in XQZ, LTH, Kitaake and Nipponbare (Nip). The samples were collected from roots of 10-day-old seedlings, stems, flag leaves, hulls, leaf sheaths, apiculi, and stigmas at heading. Data are presented as means \pm SD $(n=3)$.

Additional file 13: Table S1. Segregation of apiculi and stigma color in the $F_{2}$ population derived from a cross between Kitaake and XQZ.

Additional file 14: Table S2. Identification of tissue-specific bHLH-type genes in rice genome.

Additional file 15: Table S3. Amino acids sequences alignment of three bHLH transcription factors.

Additional file 16: Table S4. Haplotype analysis of OsPa and OsPs in 234 natural rice accessions

Additional file 17: Table S5. Promoter sequence variations of $\mathrm{OsPa}$ and OsPs in a panel of 234 natural rice accessions.

Additional file 18: Table S6. Haplotype analysis of OsC1 and OSDFR in 176 natural rice accessions.

Additional file 19: Table S7. Summary of the OsC1, OsDFR, OsPa and OsPs haplotypes in 176 natural rice accessions.

Additional file 20: Table S8. Analysis of haplotype combinations of the OsC1, OsDFR, OsPa and OsPs genes in lines with apiculus and stigma colors

Additional file 21: Table S9. Analysis of OSPAC1 coding region in a panel of 176 natural rice accessions.

Additional file 22: Table S10. Sequences of primers used in this study.

\section{Acknowledgements}

We thank Dr. Longzhi Han, Institute of Crop Sciences, CAAS, Dr. Zichao Li, Chinese Agricultural University, and Dr. Xinghua Wei, China National Rice Research Institute for kindly providing the rice mini-core collection and black rice varieties. This work was supported by grants from " 973 " project 
(2013CB127006) and the Agricultural Science and Technology Innovation Program of CAAS "Crop Functional Genomics".

\section{Authors' Contributions}

JW supervised the project; $C L, J W$ and $Z W$ designed the research; $M L, C Q$ CW, SW, and CZ performed research; YR, ZC, XZ, XG, ZZ, JW, QL and SZ provided technical assistance; $C L$ and $L M$ wrote, $H W$ and JW revised the manuscript. The author(s) read and approved the final manuscript.

\section{Funding}

The "973" project (2013CB127006) and the Agricultural Science and

Technology Innovation Program of CAAS "Crop Functional Genomics".

\section{Availability of Data and Materials}

The datasets supporting the conclusions of this article are included within the article and its additional files.

\section{Declarations}

\section{Ethics Approval and Consent to Participate}

Not applicable.

\section{Consent for Publication}

Not applicable.

\section{Competing Interests}

The authors declare that they have no competing interests.

\section{Author details}

${ }^{1}$ Institute of Crop Sciences, Chinese Academy of Agriculture Sciences/ National Key Facility for Crop Gene Resources and Genetic Improvement, Beijing 100081, China. ${ }^{2}$ State Key Laboratory of Crop Stress Biology for Arid Areas, College of Agronomy, Northwest A\&F University, Yangling 712100, Shaanxi, China. ${ }^{3}$ Key Laboratory of Crop Genetics and Germplasm Enhancement/Jiangsu Provincial Center of Plant Gene Engineering, Nanjing Agricultural University, Nanjing 210095, Jiangsu, China.

Received: 6 December 2020 Accepted: 6 April 2021

Published online: 21 April 2021

\section{References}

Albert NW, Davies KM, Lewis DH, Zhang H, Montefiori M, Brendolise C, Boase MR, $\mathrm{Ngo} \mathrm{H}$, Jameson PE, Schwinna KE (2014) A conserved network of transcriptional activators and repressors regulates anthocyanin pigmentation in Eudicots. Plant Cell 26(3):962-980. https://doi.org/10.1105/tpc.113.122069

Chen H, Zou Y, Shang Y, Lin H, Wang Y, Cai R, Tang X, Zhou J (2008) Firefly luciferase complementation imaging assay for protein-protein interactions in plants. Plant Physiol 146(2):368-376. https://doi.org/10.1104/pp.107.111740

Chen Z, Deng W, Li F, Zhou J, Li J, Xu P, Deng X, Hu F, Wang L, Chen S, Tao D (2010) A genetic study on the purple stigma genes and their locations in Oryza longistaminata. J Yunnan Univ 32:103-107 (in Chinese with English abstract)

Chin H, Wu Y, Hour A, Hong C, Lin Y (2016) Genetic and evolutionary analysis of purple leaf sheath in rice. Rice 9(1):8. https://doi.org/10.1186/s12284-016$0080-y$

Druka A, Kudrna D, Rostoks N, Brueggeman R, von Wettstein D, Kleinhofs A (2003) Chalcone isomerase gene from rice (Oryza sativa) and barley (Hordeum vulgare): physical, genetic and mutation mapping. Gene 302(1-2): 171-178. https://doi.org/10.1016/S0378-1119(02)01105-8

Duan E, Wang Y, Li X, Lin Q, Zhang T, Wang Y, Zhou C, Zhang H, Jiang L, Wang J, Lei C, Zhang X, Guo X, Wang H, Wan J (2019) OsSHI1 regulates plant architecture through modulating the transcriptional activity of IPA1 in rice. Plant Cell 31(5):1026-1042. https://doi.org/10.1105/tpc.19.00023

Fan F, Fan Y, Du J, Zhuang (2008) Fine mapping of C (chromogen for anthocyanin) gene in rice. Rice Sci 15(1):1-6. https://doi.org/10.1016/S16726308(08)60012-8

Furukawa T, Maekawa M, Oki T, Suda I, lida S, Shimada H, Takamure I, Kadowaki K (2006) The $R c$ and $R d$ genes are involved in proanthocyanidin synthesis in rice pericarp. Plant J 49(1):91-102. https://doi.org/10.1111/j.1365-313X.2006.02 958.x
Goff SA, Cone KC, Chandler VL (1992) Functional analysis of the transcriptional activator encoded by the maize $B$ gene: evidence for a direct functional interaction between two classes of regulatory proteins. Genes Dev 6(5):864875

Goff SA, Klein TM, Roth BA, Fromm ME, Cone KC, Radicella JP, Chandler VL (1990) Transactivation of anthocyanin biosynthetic genes following transfer of $B$ regulatory genes into maize tissues. EMBO J 8:2517-2522

Han L, Zhang T, Xu J, Li Y, Wang X, Wu X (2006) Genetic analysis and gene mapping of purple stigma in rice. Acta Gene $\operatorname{Sin} 33(7): 642-646$. https://doi. org/10.1016/S0379-4172(06)60094-2

$\mathrm{Hu} J$ J, Anderson B, Wessler SR (1996) Isolation and characterization of rice $R$ genes: evidence for distinct evolutionary paths in rice and maize. Genetics 142(3):1021-1031. https://doi.org/10.1093/genetics/142.3.1021

Hu J, Reddy VS, Wessler SR (2000) The rice $R$ gene family: two distinct subfamilies containing several miniature inverted-repeat transposable elements. Plant Mol Biol 42(5):667-678. https://doi.org/10.1023/A:1006355510883

Kondo A (1963) Fundamental studies on rice breeding through hybridization between Japanese and foreign varieties. VII Identification of the gene system controlling anthocyanin coloration in Japanese and foreign varieties Jap. J Breeding 13:92-98

Lin-Wang K, Bolitho K, Grafton K, Kortstee A, Karunairetnam S, McGhie TK, Espley RV, Hellens RP, Allan AC (2010) An R2R3 MYB transcription factor associated with regulation of the anthocyanin biosynthetic pathway in Rosaceae. BMC Plant Biol 10(1):50. https://doi.org/10.1186/1471-2229-10-50

Ma J, Wang Y, Ma X, Meng L, Jing R, Wang F, Wang S, Cheng Z, Zhang X, Jiang L, Wang J, Wang J, Zhang Z, Guo X, Lin Q, Wu F, Zhu S, Wu C, Ren Y, Lei C, Zhai H, Wan J (2019) Disruption of gene SPL35, encoding a novel CUE domain-containing protein, leads to cell death and enhanced disease response in rice. Plant Biotechnol J 17(8):1679-1693. https://doi.org/10.1111/ pbi.13093

Mehrtens F, Kranz H, Bednarek P, Weisshaar B (2005) The Arabidopsis transcription factor MYB12 is a flavonol-specific regulator of phenylpropanoid biosynthesis. Plant Physiol 138(2):1083-1096. https://doi.org/10.1104/pp.104 058032

Montefiori M, Brendolise C, Dare AP, Lin-Wang K, Davies KM, Hellens RP, Allan AC (2015) In the Solanaceae, a hierarchy of bHLHs confer distinct target specificity to the anthocyanin regulatory complex. J Exp Bot 66(5):1427-1436. https://doi.org/10.1093/jxb/eru494

Nagao S, Takahashi M (1956) Genetical studies on rice plant. XXX The third gene in apiculus coloration. Jap J Botany 15:141-151

Nagao S, Takahashi M (1963) Trial construction of twelve linkage groups in Japanese rice. J Fac Agr Hokkaido Univ 53:72-130

Naing AH, Kim CK (2018) Roles of R2R3-MYB transcription factors in transcriptional regulation of anthocyanin biosynthesis in horticultural plants. Plant Mol Biol 98(1-2):1-18. https://doi.org/10.1007/s11103-018-0771-4

Oikawa T, Maeda H, Oguchi T, Yamaguchi T, Tanabe N, Ebana K, Yano M, Ebitani T, Izawa T (2015) The birth of a black rice gene and its local spread by introgression. Plant Cell 27(9):2401-2414. https://doi.org/10.11 05/tpc.15.00310

Oka HI (1991) Analysis of genes for stigma coloration in rice. In: International rice research institute, eds. Rice Genetics II, Manila, pp 97-110

Oshima M, Taniguchi Y, Akasaka M, Abe K, Ichikawa H, Tabei Y, Tanaka J (2019) Development of a visible marker trait based on leaf sheath-specific anthocyanin pigmentation applicable to various genotypes in rice. Breed Sci 69(2):244-254. https://doi.org/10.1270/jsbbs.18151

Petroni K, Tonelli C (2011) Recent advances on the regulation of anthocyanin synthesis in reproductive organs. Plant Sci 181(3):219-229. https://doi.org/1 0.1016/j.plantsci.2011.05.009

Reddy AM, Reddy VS, Scheffler BE, Wienand U, Reddy AR (2007) Novel transgenic rice overexpressing anthocyanidin synthase accumulates a mixture of flavonoids leading to an increased antioxidant potential. Metab Eng 9(1):95111. https://doi.org/10.1016/j.ymben.2006.09.003

Reddy AR (1996) Genetics and molecular analysis of the anthocyanin pigmentation pathway in rice. In: Khush GS (ed) Rice genetics III proceedings of the third international Rice genetics symposium. International rice Research Institute, Manila, pp 341-352

Reddy VS, Scheffler BE, Wienand U, Wessler SR, Reddy AR (1998) Cloning and characterization of the rice homologue of the maize C1 anthocyanin regulatory gene. Plant Mol Biol 36:497-498

Saitoh K, Onishi K, Mikami I, Thidar K, Sano Y (2004) Allelic diversification at the C (OsC1) locus of wild and cultivated rice: nucleotide changes associated with 
phenotypes. Genetics 168(2):997-1007. https://doi.org/10.1534/genetics.103. 018390

Sakamoto W, Ohmori T, Kageyama K, Miyazaki C, Saito A, Murata M, Noda K, Maekawa M (2001) The Purple leaf (PI) locus of rice: the $P{ }^{N}$ allele has a complex organization and includes two genes encoding basic helix-loophelix proteins involved in anthocyanin biosynthesis. Plant Cell Physiol 42(9): 982-991. https://doi.org/10.1093/pcp/pce128

Sun X, Zhang Z, Chen C, Wu W, Ren N, Jiang C, Yu J, Zhao Y, Zheng X, Yang Q, Zhang H, Li J, Li Z (2018) The C-S-A gene system regulates hull pigmentation and reveals evolution of anthocyanin biosynthesis pathway in rice. J Exp Bot 69(7):1485-1498. https://doi.org/10.1093/jxb/ery001

Takahashi M (1957) Analysis on apiculus color genes essential to anthocyanin coloration in rice. J Fac Agr Hokkaido Univ 50:266-362

Takahashi M (1964) Linkage group and gene schemes of some striking morphological characters in Japanese rice. In: International Rice Research Institute (ed) Rice genetics and Cytogenetics. Elservier Publishing Company, Amsterdam, pp 213-236

Takahashi M (1982) Gene analysis and its related problems. J Fac Agr Hokkaido Univ 61:91-142

Waadt B, Kudla J (2008) In planta visualization of protein interactions using bimolecular fluorescence complementation (BiFC). Cold Spring Harb Protoc 2008(5). https://doi.org/10.1101/pdb.prot4995

Wang S, Lei C, Wang J, Ma J, Tang S, Wang C, Zhao Z, Tian P, Zhang H, Qi C, Cheng Z, Zhang X, Guo X, Liu L, Wu C, Wan J (2017) SPL33, encoding an eEF1A-like protein, negatively regulates cell death and defense responses in rice. J Exp Bot 68(5):899-913. https://doi.org/10.1093/jxb/erx001

Xu W, Dubos C, Lepiniec L (2015) Transcriptional control of flavonoid biosynthesis by Myb-bHLH-WDR complexes. Trends Plant Sci 20(3):176-185. https://doi.org/10.1016/.jplants.2014.12.001

Zhang H, Gong J, Chen K, Yao W, Zhang B, Wang J, Tian S, Liu H, Wang Y, Liu Y, Du L (2020) A novel R3 MYB transcriptional repressor, MaMYBx, finely regulates anthocyanin biosynthesis in grape hyacinth. Plant Sci 298:110588. https://doi.org/10.1016/j.plantsci.2020.110588

Zhang H, Zhang D, Wang M, Sun J, Qi Y, Li J, Wei X, Han L, Qiu Z, Tang S, Li Z (2011) A core collection and mini core collection of Oryza sativa L. in China. Theor Appl Genet 122(1):49-61. https://doi.org/10.1007/s00122-010-1421-7

Zhang Y, Butelli E, Martin C (2014) Engineering anthocyanin biosynthesis in plants. Curr Opin Plant Biol 19:81-90. https://doi.org/10.1016/j.pbi.2014.05.011

Zhao S, Wang C, Ma J, Wang S, Tian P, Wang J, Cheng Z, Zhang X, Guo X, Lei C (2016) Map-based cloning and functional analysis of the chromogen gene $C$ in rice (Oryza sativa L.). J Plant Biol 59(5):496-505. https://doi.org/10.1007/ s12374-016-0227-9

Zheng J, Wu H, Zhu H, Huang C, Liu C, Chang Y, Kong Z, Zhou Z, Wang G, Lin Y, Chen $\mathrm{H}$ (2019) Determining factors, regulation system, and domestication of anthocyanin biosynthesis in rice leaves. New Phytol 223(2):705-721. https:// doi.org/10.1111/nph.15807

Zhu Q, Yu S, Zeng D, Liu H, Wang H, Yang Z, Xie X, Shen R, Tan J, Li H, Zhao X, Zhang Q, Chen Y, Guo J, Chen L, Liu Y (2017) Development of "purple endosperm Rice" by engineering anthocyanin biosynthesis in the endosperm with a high-efficiency transgene stacking system. Mol Plant 10(7):918-929. https://doi.org/10.1016/j.molp.2017.05.008

\section{Publisher's Note}

Springer Nature remains neutral with regard to jurisdictional claims in published maps and institutional affiliations.

\section{Submit your manuscript to a SpringerOpen ${ }^{\circ}$ journal and benefit from:}

- Convenient online submission

- Rigorous peer review

- Open access: articles freely available online

- High visibility within the field

- Retaining the copyright to your article

Submit your next manuscript at $\boldsymbol{\nabla}$ springeropen.com 